\title{
The interplay between oxidative stress and autophagy: focus on the development of neurological diseases
}

\author{
Marjan Talebi', Seyyed Ali Mohammadi Vadoud², Alireza Haratian' ${ }^{1}$, Mohsen Talebi $^{3,4}$, Tahereh Farkhondeh ${ }^{5,6}$, \\ Ali Mohammad Pourbagher-Shahri ${ }^{5}$ and Saeed Samarghandian ${ }^{7^{*}}$ (D)
}

\begin{abstract}
Regarding the epidemiological studies, neurological dysfunctions caused by cerebral ischemia or neurodegenerative diseases (NDDs) have been considered a pointed matter. Mount-up shreds of evidence support that both autophagy and reactive oxygen species (ROS) are involved in the commencement and progression of neurological diseases. Remarkably, oxidative stress prompted by an increase of ROS threatens cerebral integrity and improves the severity of other pathogenic agents such as mitochondrial damage in neuronal disturbances. Autophagy is anticipated as a cellular defending mode to combat cytotoxic substances and damage. The recent document proposes that the interrelation of autophagy and ROS creates a crucial function in controlling neuronal homeostasis. This review aims to overview the cross-talk among autophagy and oxidative stress and its molecular mechanisms in various neurological diseases to prepare new perceptions into a new treatment for neurological disorders. Furthermore, natural/synthetic agents entailed in modulation/regulation of this ambitious cross-talk are described.
\end{abstract}

Keywords: Autophagy, Oxidative stress, Neurological diseases, Neurodegenerative diseases, Signaling pathways, Alzheimer's disease, Parkinson's disease, Reactive Oxygen Species (ROS)

\section{Introduction}

Neurological diseases contribute to $2 \%$ of the global burden of diseases. Activation and inhibition of autophagy play a central role in neurological disorders [1]. Various types of diseases accompanying neuronal degeneration, cerebral ischemia, and neurotoxicity are examples of communal neuronal disorders with approximately high prevalence [2].

Autophagy is a Greek term consisting of "auto" that means "self" and "phage" that means "to eat" that refers to an evolutionary-conserved process [3]. Autophagy which is regarded as a catabolic pathway is a degradation

*Correspondence: samarghandians1@nums.ac.ir

${ }^{7}$ Noncommunicable Diseases Research Center, Neyshabur University of Medical Sciences, Neyshabur, Iran

Full list of author information is available at the end of the article process of intracellular organelles and proteins by lysosomes. Overall the mechanism of autophagy evolves cleansing property by removing all the degraded products and retaining the valuable component for the survival of the body [4-7]. Different regulators support and coordinate the autophagy process like the transcription factor gene etc. It also involves the homeostatic process if a change occurs due to functional genetic and over-excretion. There is a considerable accumulation of pathogenic protein in NDD, so dysfunctional autophagy occurs in this type of disease. There is a mutation in the autophagy regulation gene that induces NDD [8].

Although Autophagy is an essential mechanism used for the digestion of damaged organelles and molecules, abnormalities in this process have proved to be the onset of some critical diseases such as cancer and NDDs [9]. original author(s) and the source, provide a link to the Creative Commons licence, and indicate if changes were made. The images or other third party material in this article are included in the article's Creative Commons licence, unless indicated otherwise in a credit line to the material. If material is not included in the article's Creative Commons licence and your intended use is not permitted by statutory regulation or exceeds the permitted use, you will need to obtain permission directly from the copyright holder. To view a copy of this licence, visit http://creativecommons.org/licenses/by/4.0/. The Creative Commons Public Domain Dedication waiver (http://creativeco mmons.org/publicdomain/zero/1.0/) applies to the data made available in this article, unless otherwise stated in a credit line to the data. 
It is noteworthy to state that there are three diverse types of autophagy with reference to their modes of delivery known as chaperone-mediated autophagy (CMA, autophagy mediated by particular cytosolic proteins that encompass a pentapeptide motif), microautophagy (autophagy mediated by lysosomal action), and macroautophagy (autophagy in cells form doublemembrane vesicles, known as autophagosomes, nearby a portion of cytoplasm) [9]. Notably, the main difference between macroautophagy, chaperone-mediated autophagy, and microautophagy is that in chaperonemediated autophagy and microautophagy, direct cargo delivery to lysosomes is observed but in macroautophagy, the double-layered membrane vesicles are transferred to lysosomes. Macroautophagy engulfs accumulated proteins, long-lived proteins, pathogens, and degraded organelles with the cytoplasm. They are covered with two membranes named autophagosomes to intercalate with the lysosomes. This is degraded to recycle large molecules for reuse. Microautophagy is a process in which lysosomes engulf very few amounts of the cytosolic substrate. Chaperone-mediated autophagy is triggered from stress like starvation with heat shock cognate protein having a pentapeptide sequence containing KFERQ [10-12].

The autophagic degradation process is commenced when some cytosolic components sequester and form a cup-shaped structure termed isolation membranes. Next, the isolation membranes (also called phagophores) are elongated and finally sealed and turned into double-layered membrane vesicles called autophagosomes transferred to the lysosome for degrading steps [13].

Reactive oxygen species (ROS) have been involved in the pathogenesis of NDDs. High concentrations of ROS cause oxidative damage in the brain of patients with neurodegenerative conditions [14]. Different types of stresses affect ROS levels and, therefore, autophagy, including cellular stress, ischemia-reperfusion, hypoxia, nutritional deprivation, and exogenous substances [15].

To the best of our knowledge, neurological disease is mainly leading to incapacitated individuals. Moreover, due to the multi-faced pathology of these diseases discovery, an essential mechanism like the interplay between ROS and autophagy will be targeted in prospective therapeutic approaches [16].

We aimed to discuss autophagy and its molecular regulation, its relation with oxidative stress in neurological disorders, and a brief overview of various therapeutic implications of autophagy in the current review article. Moreover, various natural/synthetic putative therapeutic medications with the amazing potential of regulating the autophagy/ROS axis are discussed.

\section{Molecular regulation of autophagy}

The molecular mechanisms attributed to the occurrence of autophagy are widely researched. The genes playing significant roles in this process are denoted as ATG s (autophagy-related). At least there are 30 ATG genes have been identified in yeasts which there are many mammalian monologues for them. Among the ATGs genes, ATG 1-10, 12-14, 16-18, 29, and 31 (called AP$A T G$ ) are pivotal for autophagosome generation. $A P-A T G$ proteins can be divided into six types, originate on their functionality: the ATG1 protein kinase and its regulators, ATG2-ATG18 complex, the ATG8 complex, the ATG12 complex, $A T G$ 9, and phosphatidylinositol-3-kinase (ASPISK) complex. In normal homeostasis (when starvation is not applied), one of the main inhibitors of autophagy induction is the target of rapamycin (TOR) [17]. In the autophagosome formation step, the primary regulators are class III phosphatidylinositol-3-kinase (AS-PI3K) complex and ATG6. Two ubiquitin-like conjugation systems are in charge of autophagosomal membrane elongation: the ATG5 and ATG12 complex, which are localized on phagophore with ATG16, and the ATG8-phosphoethanolamine (PE) complex, which could be found on both phagophore and autophagosomal membrane [18, 19]. The ATG8-PE complex can be deconjugated by ATG4 protease in a ROS-regulated step, enabling the recycling of this protein. ATGs, AMP-activated protein kinase (AMPK) pathways, and mammalian target of rapamycin (mTOR) (have an incredible character in autophagy regulation [20].

The mTOR acts as a main effector in cerebrovascular dysfunction in AD. The mTOR is involved in brain vascular and cerebral blood flow impairment, leading to cognitive dysfunction. Additionally, mTOR induces damage in the blood-brain barrier (BBB), leading to disruption in BBB integrity and cognitive defect [20].

Unc-51-like autophagy activating kinase 1 (ULK1) is ATG1 homologous which promotes autophagy [21]. Besides, Beclin-1 is an essential protein that acted in the autophagy progression. Beclin-1 affects the autophagy process via management of vacuolar protein sorting 34 (Vps34) and generation of a complex comprising Beclin-1-Vps34-Vps15. Remarkably, Beclin-1 is not acted only in the autophagy commencement, but it also provides to maturation and biogenesis of autophagosome. Starvation is one of the well-distinguishing elicitors of autophagy, in which mTOR Complex 1 (mTORC1) is prevented. As a final point, this complex prompts phagophore creation [22-24]. Mitophagy is the best type of autophagy and is chiefly managed by microtubule-related protein 1 light chain 3 (MAP1LC3; mostly defined as LC3)-related autophagy receptors through Ub-independent and Ub-dependent pathways [25]. 


\section{The relationship between reactive oxygen species and autophagy}

ROS are a large group of highly reactive-oxygen-containing species that comprise free radicals, oxygen anions, and hydrogen peroxide and are known for their short lives and high reaction desire [26, 27]. Hydrogen peroxide is now believed to be required for insulin, cytokines, growth factors, and some signaling pathways; therefore, their regulating characteristics in biological processes are almost undeniable $[28,29]$. The metabolism of oxygen is supposed to be the main pathway for ROS production. In addition, ROS at low levels is involved in cellular signaling pathways. However, on the other hand, they can cause severe irreversible damages to proteins, DNA, and lipids at high levels of ROS existence [30]. Interestingly, a growing level of evidence proposes which ROS are witnessed as crucial signals for activation of autophagy following numerous stimuli. The high and moderate ROS amounts can precisely begin mitophagy. However, only a high amount of ROS can induce common autophagy. There is almost enough evidence that supports the relationship between excessive ROS levels being generated due to stressful conditions like starvation or hypoxia and the autophagy process [31].

Herein, the molecular signaling cascades that contributed to the accomplishment and initiation of autophagy subsequent disclosure to ROS are sophisticated. These mechanisms are mainly entailed nuclear transcriptional progress and cytoplasmic posttranscriptional progress. These transcriptional mechanisms frequently comprise the activation of p53, HIF-1, and Nrf2, then autophagy modulation occurs [32, 33]. Several studies have suggested ROS-mediated autophagy via mTOR pathways in the cytoplasm. The mTOR activation, which occurs in the presence of enough nutrients and amino acids, results in phosphorylation of ATG13, which in turn prevents the autophagic process. As a result of starvation, inhibition of mTOR leads to activation of phosphatases that increase the number of dephosphorylated ATG13 and the formation of autophagosomes [34, 35]. During nutrient deficiency, the demand for more ATP production from mitochondria is increased; therefore, more ROS are produced. It was demonstrated that hydrogen peroxide as a product of starvation-induced autophagy interacts with an essential cysteine residue located on the catalytic site of HsATG4. This cysteine is a critical point in autophagy regulation by attaching ATG8 to the autophagosomal membrane, a hallmark of the autophagic process. $\mathrm{H}_{2} \mathrm{O}_{2}$ and $\mathrm{O}_{2}{ }^{-}$produced by mitochondria are mostly available ROS to provoke autophagy. The existence of elevated levels of $\mathrm{H}_{2} \mathrm{O}_{2}$ inactivated autophagy through the PI3K/Akt signaling pathways can be reactivated by hindering PTEN and Akt or mTORC1. In the same way, excessive $\mathrm{H}_{2} \mathrm{O}_{2}$ can persuade autophagy via an AMPK-dependent gate and is along with the deterioration of mTORC1 activity. Phosphorylation of ULK1/ ATG1 conjugate by AMPK is another crucial role of this protein in the induction of autophagy in starvation conditions. Several stress response proteins, for instance, c-Jun N-terminal kinase (JNK), extracellular regulated kinase (ERK), and p38MAPK, are also concerned with inducing autophagy in the face of the high levels of ROS [13]. Notably, ATG13 phosphorylation is a primary factor in the autophagy pathways. Two serine sites in ATG13 have been identified, which in starvation condition are phosphorylated by mTOR and AMPK pathway, resulting in an autophagic response. AMPK is also a regulator for $T S C 2$, a tumor suppressor gene involved in repressing the kinase mTOR in the mTORC1 complex. In elevated levels of ROS, TSC2 is activated by a cellular damage sensor ATM (Ataxia telangiectasia mutated) through an AMPK mediated pathway which ends in mTORC1 repression and autophagy induction [36]. In a manner of accumulating these foundations, the interplay between ROS and autophagy is a double-edged sword [37].

Besides, another approach is that autophagy has been recommended as a possible survival mechanism against ROS formation by eradicating damaged or laid-off agents to avoid unnecessary oxidative injury [38]. The Keap1-Nrf2 pathway is shown as a protection pathway upon encountering oxidative stress [39, 40]. The p62 for short or $\mathrm{p} 62 / \mathrm{SQSTM} 1$ protein may involve information of autophagosome as a receptor or an autophagic adaptor. Phosphorylation of p62 in the mTORC1 autophagy signaling pathway elevates the ubiquitinated cargos and phosphorylated Keap1 integration, which is vital for degradation of the Nrf2 [41].

In summary, in a well-established review article conducted by Zhao and coworkers, six involved pathways in the diverse cell fates by the contribution of ROS generation in mitochondria. These cascades are stated as a) ROS-ATG4-LC3-II, b) ROS-TIGAR, c)ROS-Nrf2-p62, d) ROS-HIF-1-BNIP3/NIX, e) ROS-FOXO3-LC3/BNIP3, and f) ROS-AMPK-ULK1 complex [42] (Fig. 1.).

\section{Autophagy and ROS in neurological diseases}

There are many relations observed between neurodegenerative disorders and autophagy. Autophagy is dynamic for cerebral homeostasis, and whenever its regulation disturbances happen, it causes neurodegenerative effects. Generally, autophagy is vital for the differentiation, growth, maintenance, and homeostasis of neuronal cells. The aggregation of toxic and unwanted degraded molecules with pathological is vital in NDDs, comprising Parkinson's (PD) and Alzheimer's (AD) diseases. The neurodegenerative disorder is due to the activation 


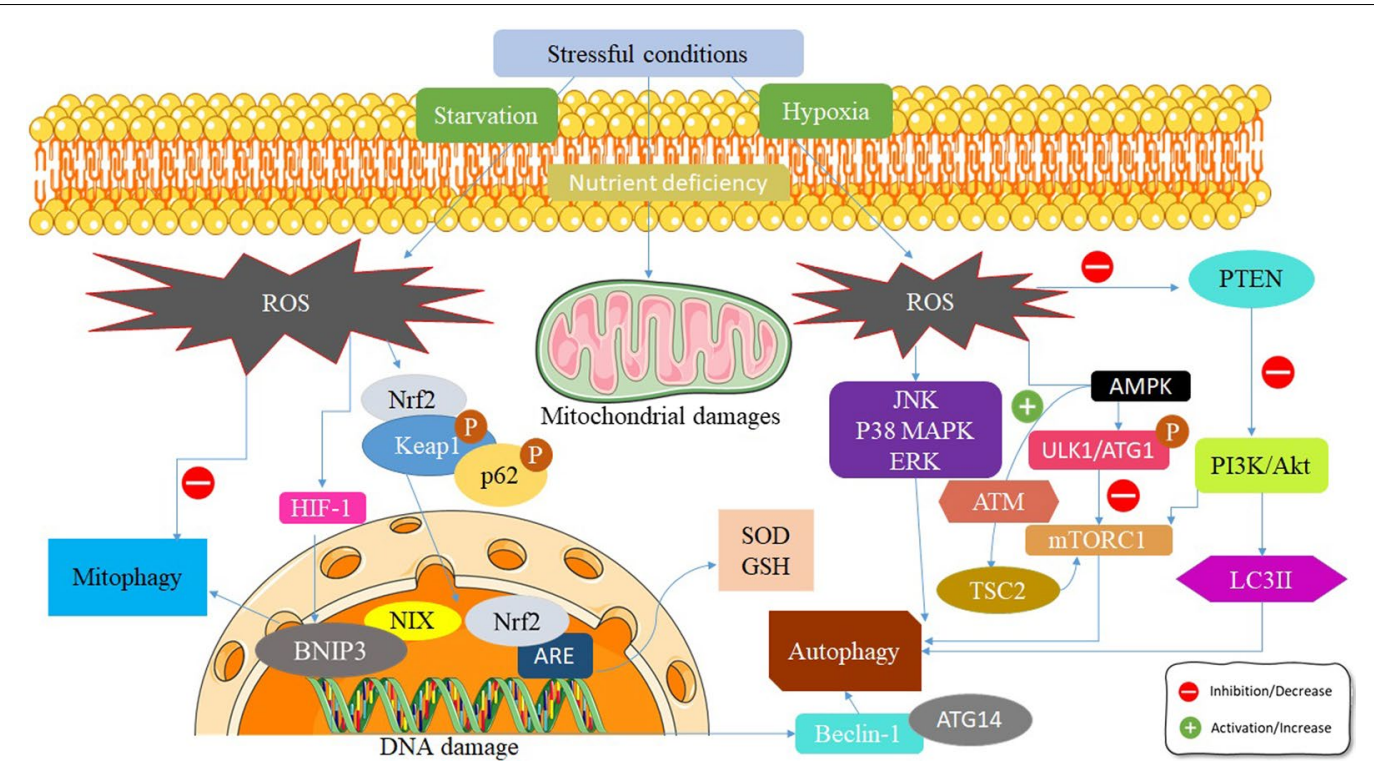

Fig. 1 The interplay between ROS and autophagy. ROS can be induced following various triggers entailing hypoxia, starvation, and mitophagy. Afterward excessive production of ROS, some factors are affected comprising HIF-1a, AMPK, JNK, and Nrf2/Keap1. Alongside these biological circumstances, autophagy may be inhibited/activated by forming the ULK complex, PI3K complex, and mTORC1 complex. Important factors involved in autophagy are LC3II and p62

of autophagy genes or autophagosomal degradation in response to stress/injury in cerebral tissues [43]. There is evidence of post-mortem brain image through electron microscopy. The irregularities in the endosomal lysosomal pathways and autophagosomal accumulation happen due to the neuronal loss by autophagy in the mouse model [44].

There are two approaches for autophagy acting via mTOR independent and dependent mechanisms. The modulation of mTOR dependent approach is through rapamycin inhibiting mTORC1 for various NDDs. The primary function of rapamycin is immune-suppressant and second as anti-proliferative property, which is causing benefit for the chronic neurodegenerative disorder. It is also observed a good response in the preclinical model. Further mTOR independent modulators for autophagy are anticipated for therapeutic efficacy by activation of AMPK molecules. Cells are not directly involved in the autophagy mechanism. They are associated with different receptors like cAMP and alpha receptors [8].

Oxidative damage is found in NDDs leading to increased autophagic activity [45]. During these conditions, autophagy cannot act as a cell-survival mechanism but stimulate cell death. In $\mathrm{AD}$ conditions, oxidative stress induces neuronal cell death via stimulating autophagy of accumulated $A \beta$ and permeabilization of the lysosomal membrane leading to neuron death. Oxidative stress-induced-mitochondrial damage in pyramidal neurons is considered as autophagic degradation in $\mathrm{AD}$ (mitophagy), resulting in neurodegeneration. Improper expression of protein phosphatase 2A (PP2A) is related to the onset of some NDDs via an increase in susceptibility to oxidative stress-mediated cell death [46].

Oxidative stress also can stimulate autophagy in neurons via downregulating Oxi- $\alpha$ (a neuroprotective protein in dopamine neurons). In a normal cell, Oxi- $\alpha$ induces mTOR and subsequently decreased autophagy. However, overproduction of ROS decreases the expression of Oxi- $\alpha$ and resulting in a decrease in mTOR activity, leading to autophagy [47].

A summary of compounds affecting autophagy in neurological diseases is stated in Table 1.

\section{Alzheimer's disease}

Alzheimer's disease is commonly well-identified as one of the most prevalent diseases contributed to neurodegeneration, hallmarked by a progressive loss of memory and cognition deficits. AD is characteristically described by the existence of extracellular amyloid $\beta$ $(\mathrm{A} \beta)$ plaques and intracellular tau $(\tau)$ protein tangles as the principal biomarkers. $A \beta$ is produced by the assistance of the gate attributed to the enzymatic cleavage of the amyloid precursor protein $(A P P)[48,49]$. Oxidative stress is a conspicuous etiological factor responsible for $\mathrm{AD}$ pathogenesis, associated with the establishment of the A $\beta$ plague, phosphorylation of $\tau$ protein, and subsequently the development of the neurofibrillary tangles 


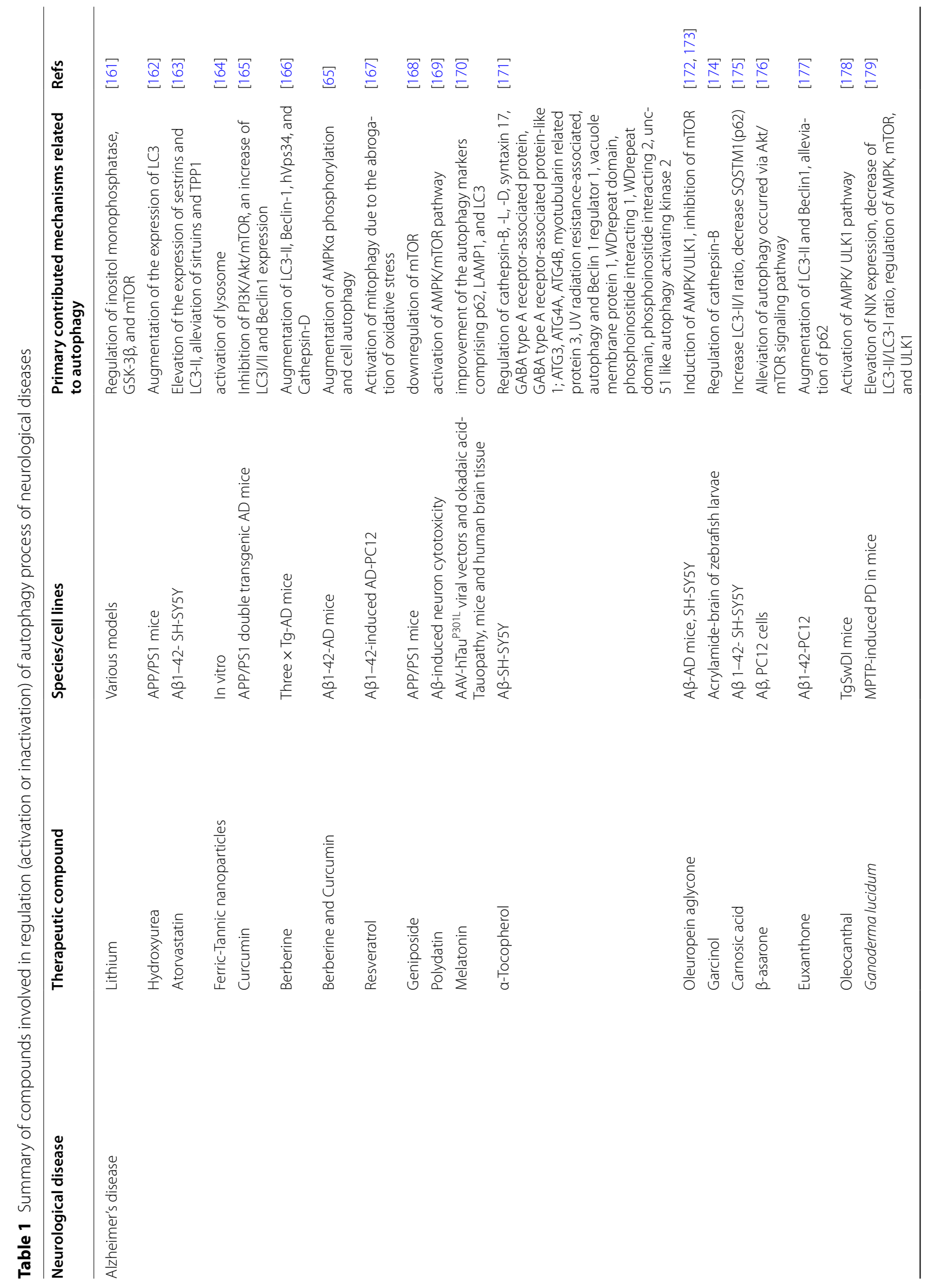




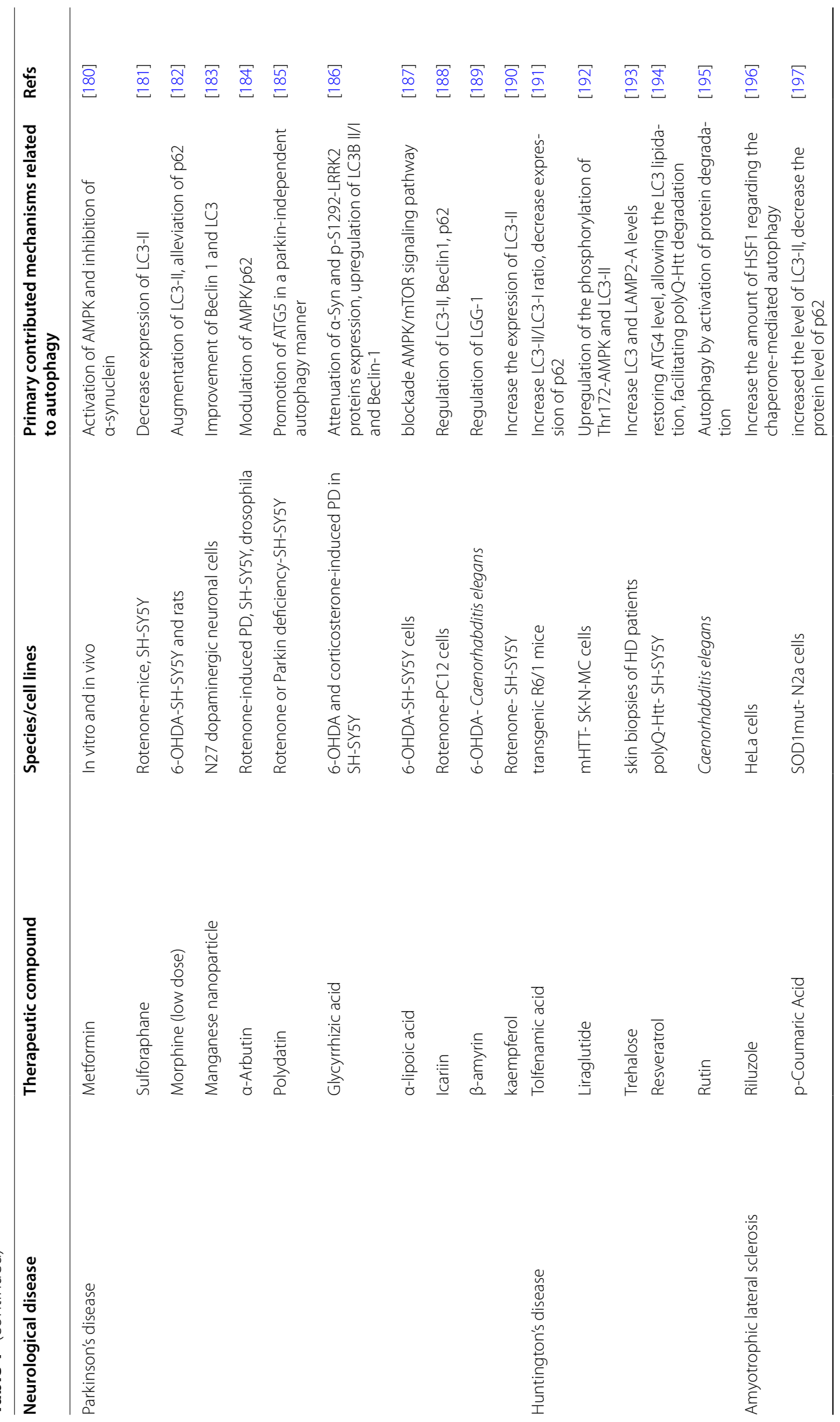




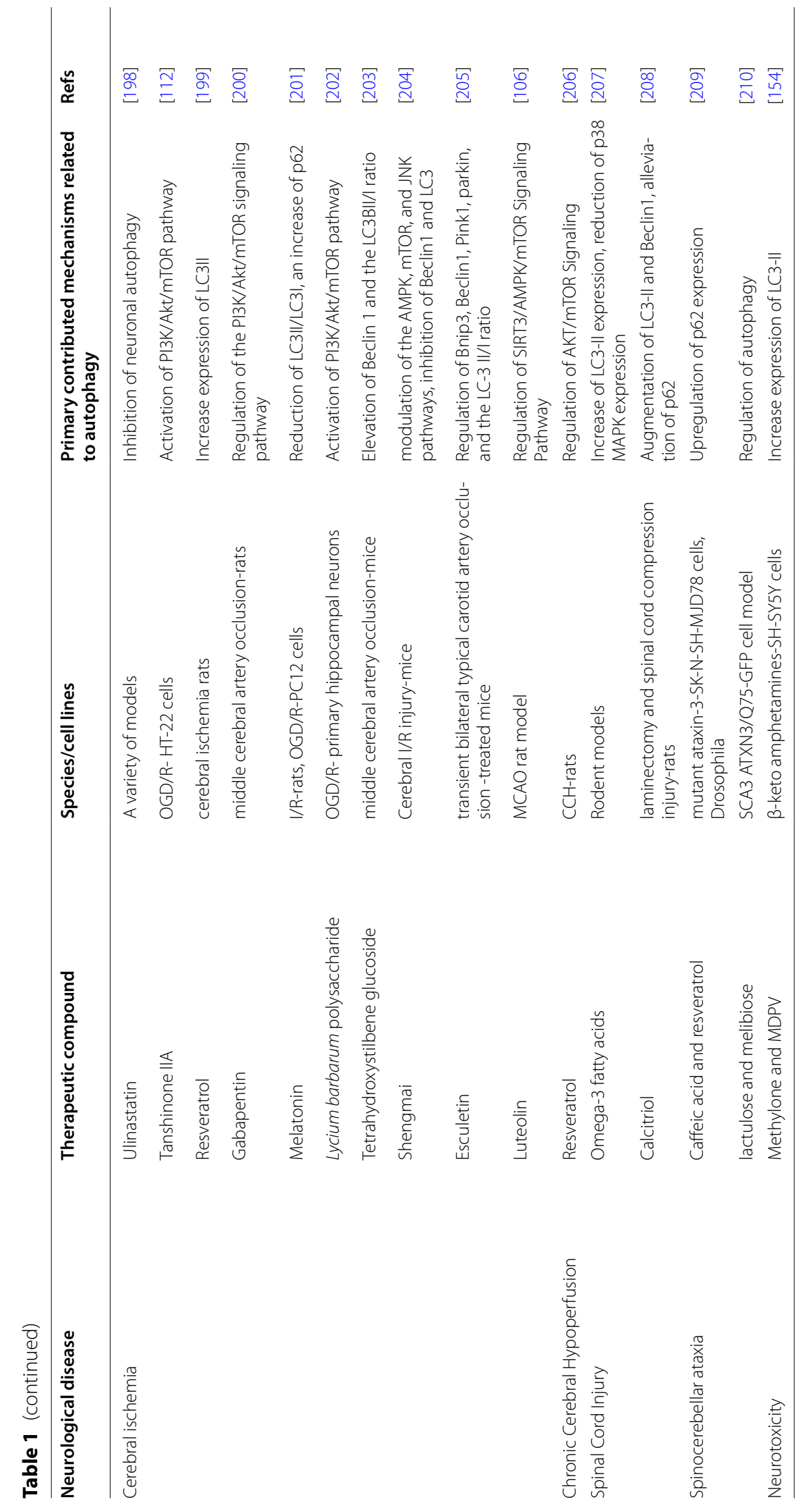


(NFTs) $[27,50]$. Dysregulation of autophagy is responsible for the accumulation of pathogenic proteins that contributed to neurodegeneration such as alterations in ubiquitinated proteins, degradation of $\mathrm{A} \beta$, and phosphorylation of $\tau$ protein. The gathering of $\mathrm{A} \beta$ leads to perceiving of diminished fusion of autophagosomes with lysosomes. Autophagy is taking part in the secretion of $\mathrm{A} \beta$ into the extracellular surroundings, wherever it fabricates the plaques. The omission of ATG7 in $A P P$ transgenic mice causes downregulation in secretion and formation of $\mathrm{A} \beta$. A mutation in Presenilin1 (PSEN1), which is entailed in cleavage of $A P P$, elucidates one of the important features related to $\mathrm{AD}$ and leads to the deficiency of lysosome functional mission and the $\mathrm{A} \beta$ accumulation. Furthermore, PSEN1 plays a part as an ER chaperone for the V0a1 subunit of lysosomal v-ATPase. The witnessed mutation damages lysosomal v-ATPase maturation and consequently surges lysosomal $\mathrm{pH}$. Hence, the accumulation of $\tau$ protein into intracellular tangles is likewise a hallmark that contributed to AD pathogenesis [51]. Hyperphosphorylated $\tau$ protein co-localizes with p62 and LC3B-II in $\mathrm{AD}$ patients and more neuronal disturbing conditions for instance corticobasal degeneration and progressive supranuclear palsy. Additionally, abnormal $\tau$ proteins induce a disturbance in axonal vesicle transport via the impeding of complex, so resulting in the elevation of amount of autophagosomes in AD [52]. As Hsc70 interrelates with $\tau$ in the monomeric, oligomeric, and aggregated arrangement and clears by the CMA pathway, autophagy can be selective. Lysosomeassociated membrane proteins-2A (LAMP-2A) is the crucial agent involved in CMA and interacts with the Hsc70- $\tau$ complex and activates the downstream cascade. Besides, chronic stress or environmental risk factors and augmented glucocorticoid levels have the capability to simulate AD and other coherent dysfunctions. Augmentation in levels of glucocorticoid may possibly enable the $\mathrm{A} \beta$ plague formation and $\tau$ protein phosphorylation which can be mediated by mTORreliant impeding of autophagy [53-56]. Thus, it is also linked to glucocorticoid-persuaded oxidative stress. By considering recent research works, it is now comprehended that autophagy change and stress-related constituents can feasibly be managed by numerous RNA binding proteins (RBPs) such as DEAD-box 5 (DDX5), fused in sarcoma (FUS), Ras GTPase-activating protein-binding protein 1 (G3BP1), poly (A)-binding protein (PABP), and T-cell intracellular antigen 1 (TIA-1). The level of the aforementioned proteins is raised in response to chronic stress and encountering chronic amounts of glucocorticoid. Above and beyond, these RBPs are appeared to be interconnected to oxidative stress reactions. For that reason, they could be considered as insightful therapeutic targets for averting the development of stress granules in AD and other $\tau$ pathologies. Regarding the study mentioned above conducted by Huang and colleagues, circular RNAs (circRNAS) can regulate pathological progressions by binding to RBPs, sponging miRNAs, modulating mRNA, aiding as biomarkers, and potential therapeutic targets $\mathrm{AD}$ [57]. Lower expression of Beclin-1 was found in the brain of an AD patient also affects autophagy. Emerging evidence proposes that spatial learning and memory deficits in AD may be strongly interrelated with the deficiency of the Nrf2-ARE pathway. Amazingly, some researchers have recommended that the interaction between oxidative stress and mitochondrial dysfunction is feasibly convoluted in AD progression owing to the impact of oxidative stress on mitochondrial transport [54]. The autophagic elimination of impaired mitochondria and $\mathrm{A} \beta$ mediated by Parkin can diminish oxidative stress and reinstate the energy source to delay or avoid neurodegeneration in AD transgenic mice [58]. $\mathrm{Du}$ and coworkers presented a study focused on gene therapy-mediated PINK1 overexpression, which could enhance the clearance of impaired mitochondria via increasing activation of autophagy receptors containing OPTN and NDP52, which in this manner led to alleviation of $\mathrm{A} \beta$-induced synaptic loss and cognitive deficit in AD mice [59]. Li and colleagues hypothesized that mTOR might mediate glucagon-like peptide-1 therapy in $\mathrm{AD}[60]$.

It has been observed that ATG12 and ATG5, Beclin1, and LC3-II increased considerably, whereas p62 expression reduced in the $\tau$ hyperphosphorylation rodent model of AD [61].

Activation of AMPK is provocative in the deposition of $\mathrm{A} \beta$ and phosphorylation of $\tau$; however, it can ameliorate autophagy, restore mitochondrial health, mitigating insulin resistance, and abrogate oxidative damage [62].

Zhang and colleagues found that overexpression of transcriptional factor EB (TFEB) attenuated AD progression by decreasing the accumulation of $A \beta$ via regulation of the autophagy-lysosome pathway entailing LAMP-1, cathepsin D, LC3-II, and p62 and alleviating A $\beta$-induced ROS generation and cell fate [63]. Diminishing the p62 protein level can disturb Nrf2, cyclic AMP, and NF- $\mathrm{kB}$ signaling pathways, increasing oxidative stress during $\mathrm{AD}$ [21]. Omata and coworkers discussed that expression of some autophagy-related genes such as ATG1, ATG8A, and $A T G 18$ is regulated by foxo/sir2-mediated aging procedures in AD [64].

Trichostatin A (histone deacetylase inhibitor) improved the expression of $\mathrm{Nrf2}$ and its downstream genes, thus 


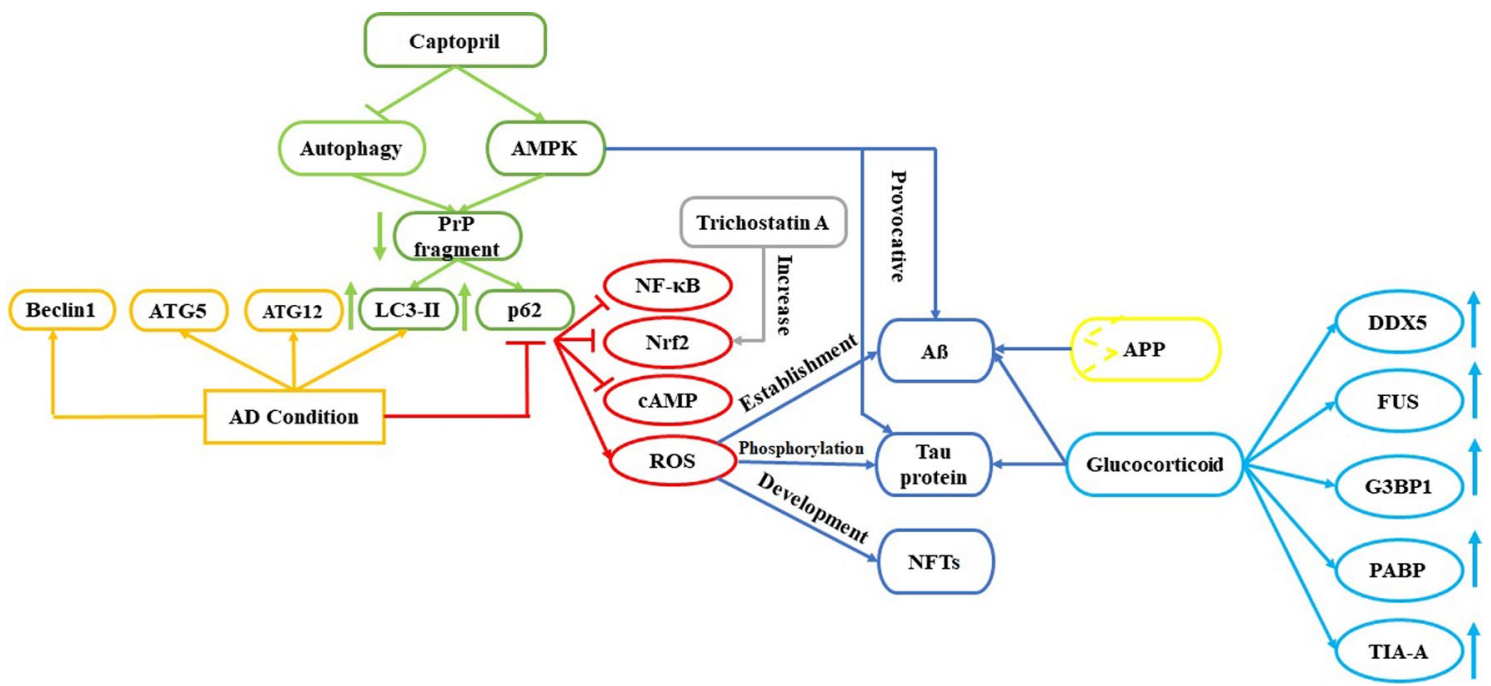

Fig. 2 The interplay between autophagy and oxidative stress in Alzheimer's disease. AD condiiton is acompained by increase in $L C$ and inhibition of p62 expersions that inhibit the NFKB, Nrf2 and CAMP but increase ROS production

augmenting the total antioxidant capacity of SH-SY5Y cells and hindering $\mathrm{A} \beta$ peptide-induced autophagy [65].

$\mathrm{AD}$ condition is accompanied by increase in the expression of beclin 1, ATG5, ATG12, LC3-II and p62.The effect of autophagy regulation by captopril and its effect on reducing a neurotoxic effect of prion peptide $(\mathrm{PrP})$ that exerts its neurotoxic effects through changes in calcium metabolism has been explored. Captopril reduced PrP Fragment 106-126 neurotoxicity cell death by activating AMPK and blockage of autophagy, which elevated the proteins LC3-II and p62. Stimulation of LC3-II and p62 expressions could inhibit the expression of NFKB, Nrf2 and cAMP and increase ROS production resulted in $A B$ formation, phosphorylation of tau protein and NFTs formation. They suggested that AMPK is a crucial pathway of captopril in autophagy regulation and a possible choice for investigation in Alzheimer's disease [19, 66, 67].

Weng and coworkers elucidated that the administration of camellia oil might alleviate AD pathogenesis through the microbiome-gut-brain axis and regulation of oxidative stress, autophagy, and inflammation [68] (Fig. 2.).

\section{Parkinson's disease}

Parkinson's disease (PD) is a movement disorder with remaining clinical features including resting tremor, bradykinesia, rigidity, and postural instability. Based on some study some pieces of evidence display autophagy dysfunction and oxidative stress, implicated in the pathogenesis of PD, which leads to steadily loss of dopaminergic neurons in the Substantia Nigra. Autophagy is essential for the differentiation, growth, maintenance, and homeostasis of neuronal cells $[69,70]$.
Modulations of the expression of BECN1 and TFEB as autophagy-related genes and regulation of numerous autophagy regulators, for instance, rapamycin, trehalose, lysosome modulators, etc., have exerted anti-PD implications in a variety of experimental models [71].

A number of genes are correlated to the primary pathology of PD, encompassing $\alpha$-synuclein, GBA, Parkin, and PINK1. Autosomal recessive PD is connected to the taking place of mutations in PINK1 and Parkin, which motivate damage in the degradation of injured mitochondria through activation of mitophagy [54]. Genetic erosion of Pink1 led to a deficiency of striatal mitochondria respiration and weakness to oxidative damage in the neuronal cells [72]. Likewise, the ablation of Parkin resulted in dysfunctions that were attributable to synaptic plasticity and striatal mitochondria [73]. PD is also described by Lewy bodies in the neuronal nucleus, which contains protein aggregate of $\alpha$-synuclein which are regarded as insoluble and degraded by CMA. Though, mutant $\alpha$-synuclein has a great affinity with LAMP-2A, which hinders lysosomal uptake of the substrates, therefore stopping CMA-dependent degradation. Self-determining of the protein attachments, an amplified $\alpha$-synuclein level damages autophagy, which results in ATG9 mislocalization [74].

The $\alpha$-synuclein is involved in the cytoplasmic clearance in autophagy responsible for Parkinsonism. The recent advancement in the study suggested that a generation of pathological abnormalities due to Beclin-1 overexpression causes low clearance of $\alpha$-synuclein [75].

Mitochondria have a vital function in redox regulation of autophagy as the producer and scavenger of ROS. The 
dysfunction of mitochondria is a noticeable commencing factor of nervous system diseases which can be initiated by exceeding ROS. Mitophagy is considered a strategy for clearing the impaired mitochondria to restore homeostasis substantial pathological stress $[25,75]$.

Dagda et al. revealed that knockdown of PINK1 in a recessive PD model could cause the accumulation of mitochondrial ROS in consort with clustered fragmented/depolarized mitochondria associated with autophagy [73]. Furthermore, it is noteworthy to discuss the crucial function of autophagy in the limitation of neuronal fate induced by RNAi knockdown of genes engaged in autophagy regarded PD [76].

Long noncoding RNAs (lncRNAs) have a prospective and versatile role in regulating autophagy engaged in PD by the mediation of diverse targets such as PTEN1, mTOR, etc. TFEB-mediated autophagy is a potent mechanism for survival throughout oxidative stress in PD. Zhuang and coworkers obtained valuable findings, including autophagy enhancing impacts of Torin1 (an mTOR-dependent TFEB/autophagy promoter) and curcumin analog C1 (a TFEB-dependent and mTOR-independent autophagy promoter) in PD models [77].

Ning and coworkers demonstrated that $\beta$-asarone might regulate the ER stress-autophagy by impeding the PERK/CHOP/Bcl-2/Beclin-1 signaling pathway in 6-OHDA-induced parkinsonian rats [73, 78]. Numerous studies have indicated that mitochondrial dysfunction and mitochondrial complex I deficiencies also contribute to PD by aggregating oxidative damage in dopaminergic neurons, which can play a role in the initiation or progression of this disease. DJ-1, a contributing protein in the incidence of familial PD, is indispensable for moderating PINK/Parkin-mediated mitophagy. DJ-1 and DJ-1-binding complexes have been recognized for neuroprotection combat oxidative stress in PD rats $[12,79]$.

1-methyl-4-phenyl-1, 2, 3, 6-tetrahydropyridine (MPTP) by reduction of tyrosine hydroxylase $(\mathrm{TH})$ protein and dopamine (DA) amount creates a PD model in an animal model. Furthermore, Ebert and colleague's study exhibited that Insulin-like growth factor-1 (IGF-1) had growth effects on deteriorating dopamine-producing neurons in a PD rat model and assist the durability of human neural progenitor cells (hNPC) in vitro and post transferring to rat PD model [80].

Exogenous IGF-1 as a neuroprotective agent has shown the potential to reduce neuronal lesions. In a study WenFang and colleagues explored the IGF-1 effect on MPTP/ MPP + induced neuronal damage. IGF-1 could downregulate autophagy by hindering signaling of the PI3kAkt-mTOR pathway and activation of G-protein coupled estrogen receptor (GPER). However, they indicated that more study is needed to understand this mechanism of action of IGF-1 in the CNS [81].

Apelin-36 as a neuroendocrine peptide combated MPTP-induced PD in mice that might be connected with mitigation of oxidative stress and the amelioration of LC3-II and Beclin1 and inhibition of p62 expression (82). Glucagon-like peptide-1, -2 protected neuronal cells in PD circumstance via improving ATG7, LC3B, and Beclin1 [83]. Poly (ADP-ribose) polymerase 1 (PARP1) signaling pathway, which is a regulator for oxidative stress, can regulate TFEB-mediated autophagy as a therapeutic approach to combat PD via amelioration of $\alpha$-synuclein degradation [84].

Lactulose and melibiose upregulated levels of SOD2, Nrf2, and NQO1 regarding the oxidative stress process and improved the LC3-II/LC3-I ratio in the association of autophagy in the MPTP-ventral midbrain [85]. Guo et al. discovered that GM1 ganglioside has an anti-PD potential which is described as the ability to promote autophagy by the autophagosomes in the substantia nigra of PD mice along with alterations of LC3-II and p62 in the MPP +-treated SH-SY5Y cells, which are dependent on the removal of $\alpha$-synuclein [86].

\section{Huntington's disease}

Huntington's disease (HD) is a neurodegenerative disorder instigated by mutant proteins accompanying extended glutamine replications (polyQ) [87]. HD pathogenesis is intensely impacted by neuronal autophagy dysfunction. Huntingtin (HTT) is the most feasible polyQ protein for HD incidence. According to previous studies, HTT damages autophagosomes [76]. The wild type of HTT functions as a scaffold protein entailed in the signaling of several autophagy proteins to the autophagosome in the selective autophagic procedure. A loss of HTT results in the observation of failure in autophagosome transport and successively causes substrates degradation. Mutant $(\mathrm{m}) \mathrm{HTT}$ also prevents a striatum-specific protein, Rhes, which interacts with Beclin1 to process autophagy. Tsunemi et al. claimed that peroxisome proliferator-activated receptor $\gamma($ PPAR $\gamma$ ) coactivator $1 \alpha$ $(\mathrm{PGC}-1 \alpha)$, which is a regulator of oxidative stress and mitochondrial biogenesis, could improve HD hallmarks in a murine model. They discovered that PGC- $1 \alpha$ promoted HTT throughput by activation of TFEB [88].

Prolonged polyQ regions adjust BECN1 deubiquitination and decline BECN1 protein levels. Hence they can inhibit starvation-induced autophagy. In addition, polymorphisms in ATG7 are connected with the early onset of HD. Amelioration of autophagy via hindering mTOR facilitates the HTT aggregates' clearance [89]. 


\section{Amyotrophic lateral sclerosis}

Amyotrophic lateral sclerosis (ALS) is a NDD described by the occurrence of the selective fate of motor neurons. p62/SQSTM1 is a central factor responsible for the physio/pathological targeting of ALS [76]. It has been observed that C9ORF72 depletion via regulation of mTORC1, TANK-binding kinase 1 (TBK1), optineurin, valosin-contain protein, $\mathrm{Cu} / \mathrm{Zn}$ superoxide dismutase (SOD1), ALS2 (Alsin), p62, vesicle-associated membrane protein-associated protein B (VAPB or ALS8), and sigma receptor-1 (SigR1 or ALS16) have participated in ALS and frontotemporal dementia (FTD) as autophagyrelated NDDs [90, 91].

\section{Ataxia}

Fragile X-associated tremor/ataxia syndrome (FXTAS) is a neurodegenerative condition accompanying a permutation repeat expansion (55-200 CGG repeats) in the $5^{\prime}$ noncoding region of the FMR1 gene. Ma et al. reported that the existence of elevated oxidative stress led to autophagy. Indeed, the study showed that exceedingly enhanced levels of conjugated small ubiquitin-related modifier 2 (SUMO 2) protein and p62/sequestosome-1 (p62/SQSTM1) protein were observed via autofluorescence-based fluorescence-activated cell sorting (FACS) and liquid chromatography/tandem mass spectrometry (LC-MS/MS)-based proteomics within the inclusions [92].

Spinocerebellar ataxia type 2 (SCA2) is a rare polyglutamine-dependent NDD affected by a CAG repeat expansion in the ataxin-2 gene. Although in a recent study, it has been found that oligomerized ataxin-2 and oxidative stress impact autophagic clearance in SCA2 cells; this happening is involved in the pathophysiology of SCA2, activation of autophagy or clearance of oligomers can verify to be beneficial approaches in treatment [93].

Autosomal recessive spastic ataxia of CharlevoixSaguenay (ARSACS) is a sporadic early-onset neurological disease instigated by mutations in SACS, which encodes sacsin. Expression of LC3 decreased, and levels of p62 increased even after treatment with the lysosomal inhibitor bafilomycin A1, representing deficiency of the autophagic flux with correlation to oxidative stress and mitochondrial degradation [94]. Thus, the loss of sacsin is caused by oxidative stress and mitochondrial dysfunction, a novel mechanism in the pathogenesis of ARSACS [94].

\section{Spiral ganglion neuron degeneration}

Dysfunction of autophagy is a significant factor in spiral ganglion neuron (SGN) degeneration. Thus, TFEB may be a possible objective for diminishing SGN degeneration subsequent sensory epithelial cell loss which can be oxidative stress in the cochlea of mice [95].

\section{Cerebral ischemia}

L-glutamine (L-Gln), an amino acid, has a crucial intermediary role in mTORC1 activity and is a premier substance in some solute carriers (Slc), transmembrane transporters families like the Slc38a and SNAT1 families. L-Gln initiating mTORC1 signaling via the concurrent opposing flow of L-Gln and essential amino acids (EAAs) [96]. L-Gln are neuron-specific transporters and are an essential part of neurological disorders. However, their engagement in neurological disorders is still not wholly recognized and needs more studies. Sodiumcoupled neutral amino acid transporter 1 (SNAT1) upregulates mTORC1 activity, which induces neurological disorders like ischemic strokes to increase cell death. Ribosomal protein S6 kinase beta-1 (p70S6K1) regulates translation by phosphorylation of ribosomal protein $\mathrm{S} 6$ and p70S6K1itself phosphorylation by mTORC1, based on the phosphorylation level, can act as a successor for mTORC1. Comparing mRNA amounts of Slc transporters showed SNAT1 expression in the brain, particularly in neuronal nuclei (NeuN) positive neurons but not in glial cells. Inhibition of mTORC1, which initiated autophagy, improved cell protection and reduced neuronal damage in the ischemic mice model. So, because of the site-specific expression of SNAT1, this was proposed as a prospective target of subsequent therapies for neurological disorders [97-100].

Hamartin (Tsc1) augmented resistance to oxygen-glucose deprivation (OGD) and neuronal ischemia by inducing beneficial autophagy through a mTORC1-dependent mechanism [101].

Neuronal-targeted TFEB restored the impairments of the autophagy-lysosomal pathway and mitigated cerebral ischemic injury [102].

Studies on miR-497 show their intervention in the pathological process of CNS diseases, but the exact pathway is not entirely understood. Kunlin Jin and colleagues explored miR-497 agonist (agomir), the miR-497 antagonist (antagomir), and 3-Methyladenine (3-MA), as an autophagy inhibitor, in a rat model of cerebral ischemic attack. They reported that treatment with miR-497 antagonists decreased the infarction zone, altered ischemic stroke neuronal decay, and increased LC3 expression. Nevertheless, when 3-MA was used for treating ischemia in rats, opposing the miR-497 antagonist, inhibition of autophagy did not show neuroprotective effects and infarction zone reduction. The miR-497 antagonist could improve cerebral recovery by upregulating autophagy in an age-dependent order. Moreover, the efficacy of miR497 antagonist in cerebral recovery and infarction zone 
reduction was less effective in older rats than in younger rats $[76,103]$.

Regarding the shreds of evidence reflected in published documents, venous thrombolysis and endovascular intervention are usual therapeutic strategies used for reestablishing the blood supply obligatory to improve nerve tasks in ischemic cerebrovascular disease. Nevertheless, together animal and clinical studies have publicized that reperfusion subsequent ischemia leads to surveillance of brain damage with more severity. The ischemia-reperfusion injury (IRI) may be characterized as a problematical pathologic condition encompassing numerous factors such as oxidative stress. Extensive damages attributed to mitochondria have been considered in IRI containing unbalanced swelling and crista fragmenting of mitochondria, in ischemic cerebrovascular disease, particularly throughout the acute phase. This impairment motivates mPTP to open unremittingly, leading to the occurrence of an alteration in ROS formation, energy shortage, and membrane potential, thus prompting autophagy. It has been witnessed that autophagy is caused in the mouse striatum and cortex subsequent cerebral hypoxicischemia and augmented by a consequent ROS overproduction. Oxidative damage in mouse striatum and cortex subsequent cerebral hypoxia-ischemia causes autophagy. Autophagy during this circumstance can noticeably save neurons in the ischemic condition by inhibiting necrosis and apoptosis via abolishing damaged mitochondria [104].

It has been conveyed that insufficiency of fatty acids (FAs) intensely alters ischemia-induced autophagy activation. This is revealed by the raised levels of LC3 and Beclin1 expression, along with significant growth in 8-OHdG, signifying that FAs deficiency can ameliorate the levels of autophagy via induction of oxidative damage. A study has presented that together ROS and autophagy are involved in reperfusion injury afterward cerebral ischemia which antioxidants frequently stimulate autophagy [104]. The use of antioxidants well-identified as revulsive of autophagy has the ability to attenuate neuronal damage and expressively alleviate the infarcted area. Therefore, we will wonder that antioxidants might play a defensive character in ischemic injury by prompting autophagy. There could also be some more complex crosstalk mechanisms concerning autophagy and oxidative stress in the necessity of additional research [105].

Furthermore, SIRT3 may be a well-maintained deacetylase connected to biological roles like stress resistance, mitochondrial redox homeostasis, and energy metabolism. Furthermore, it can positively regulate autophagy over the AMPK-mTOR pathway, which endorses neuronal persistence in an in vitro oxygen and glucose deprivation (OGD) model of cerebral ischemia made by decreasing $\mathrm{H}_{2} \mathrm{O}_{2}$ and $\mathrm{O}_{2}{ }^{-}$. Pharmacological or genetic impeding of autophagy can amend SIRT6-mediated neuronal damage, feasibly via mitigating Akt signaling allied with oxidative damage in the OGD model of SHSY5Y neurons. Besides, moderate activation of ROS can encourage Parkin translocation into the injured mitochondria, afterward suffer Parkin-mediated mitophagy and certify the mitochondria integrity in ischemic brain injury [106].

\section{Chronic cerebral hypoperfusion}

Reduced blood flow is linked to many CNS diseases, like Alzheimer's disease, atherosclerosis, and cerebral small vessel disease. So, as a blood flow reduction situation, chronic cerebral hypoperfusion is a problem for treating many debilitating cerebral disorders, leading to apoptotic neuronal cell death and cognitive disorders. Hence, for decreasing apoptotic neuronal cell death, a treatment for chronic cerebral hypoperfusion could be a good idea [76].

$\mathrm{Su}$ and colleagues explored cannabinoid receptor agonist WIN55,212-2 (WIN) and fatty acid amide hydrolase inhibitor URB597 (URB) in a rat model to be considered for treating chronic cerebral hypoperfusion induced apoptosis. They found that WIN and URB treated rats showing better action in memory tests and neural cells counting, proteomics, genomics, and brain sections analysis showed a reduction of neurotoxicity, phosphorylation of c-Jun $\mathrm{N}$-terminal kinases (JNK), caspase-3 initiation, and Bcl-2 to Bax ratio [107-109]. In another study by this team, they investigated the URB effect on up-regulated autophagy in chronic cerebral hypoperfusion status in the brains of a rat model of chronic cerebral hypoperfusion; they reported that inhibition of autophagy by URB reduced autophagosomes accumulation and decreased synaptic degradation caused by excessive autophagy, and diminished mitochondrial dysfunction and mitophagy. Based on increasing phosphorylated $A k t$ and mTOR levels and unchanged AMPK, they suggested Akt-mTOR signaling as a possible pathway to inhibit autophagy by URB [110].

\section{Spinal cord injury}

A meta-analysis about the effect of autophagy regulation on spinal cord injury improvement showed it altered neurological recovery, whether it is increased or decreased, but no apparent discrepancy was seen among increment and decrement of autophagy in spinal damage treatment in a rat model [111].

Zhu et al. discussed that acupuncture could increase mTORC1expression in the peri-infarct cortex and decrease ULK1, ATG13, and Beclin1 amounts. However, above and beyond, acupuncture attenuated LC3-II and 


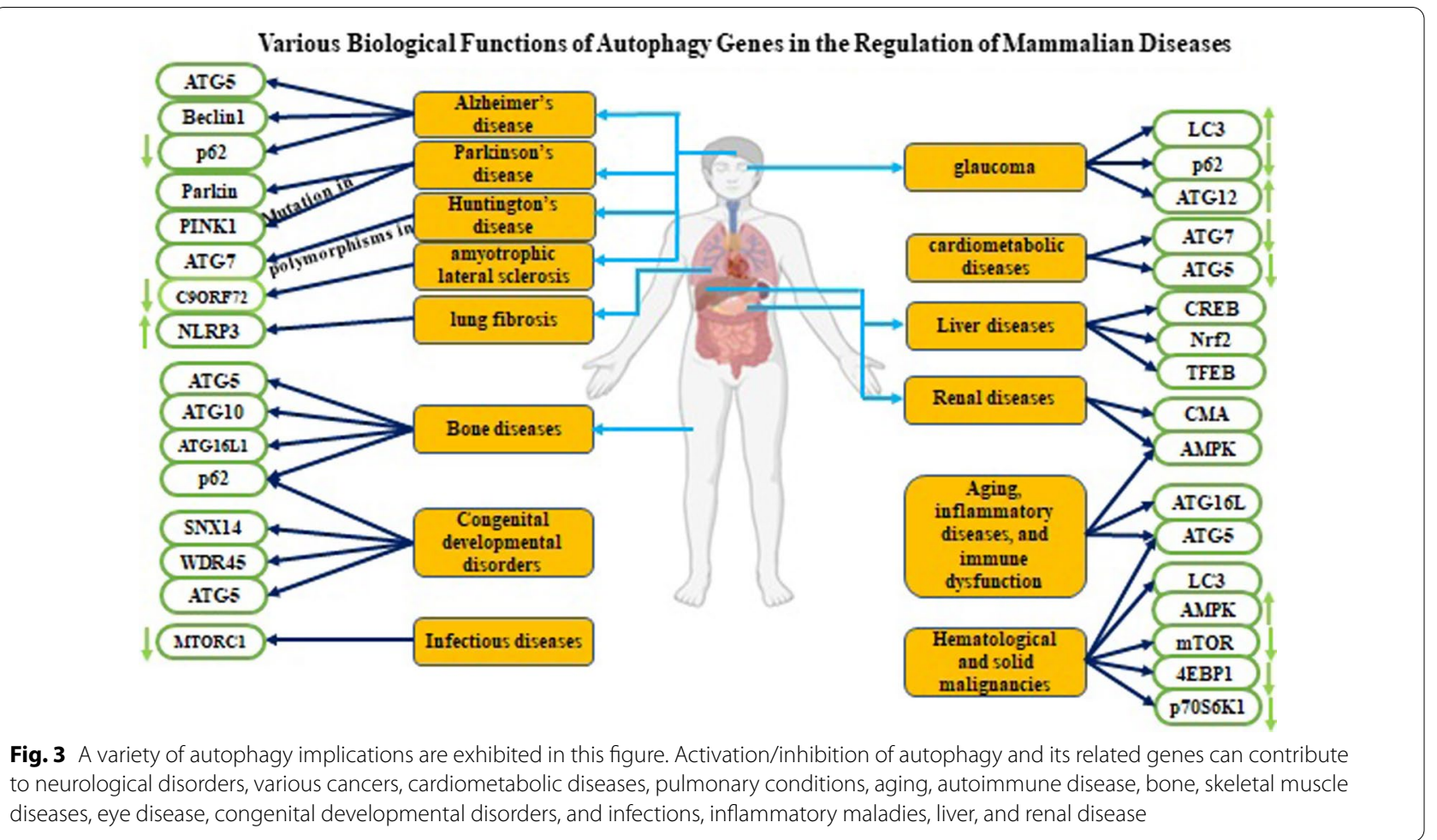

Beclin 1 expression and the number of autophagosomes [112].

Transcription factor E3 (TFE3) has excellent potential for use in ROS-mediated autophagy dysfunction following spinal cord injury. TFE3 has been regulated partially afterward the spinal cord injury via regulation of AMPKmTOR and AMPK-SKP2-CARM1 signaling pathways [61].

\section{Therapeutic implications of autophagy dysregulation}

The induction of therapeutic autophagy acts as a survival mechanism. However, progressive autophagy, also known as autophagic cell death, can lead to non-apoptotic cell death. Many human diseases such as autoimmune diseases, cancers, cardiovascular disorders, microbial infections, metabolic diseases, inflammatory responses, bone diseases, renal ailments, liver disorders, NDDs, etc., have been studied. Autophagy can be utilized both in maladaptive and adaptive functions in the pathogenesis of various diseases (Fig. 3.) [113].

\section{Microbial and viral infections, and parasites}

Khandia and coworkers explained the roles of autophagy in infectious diseases in their recent review article very carefully. Autophagy has a critical role in viral infections, for instance, bird flu, swine fever, Ebola virus disease,
Zika virus infection, SARS, Chikungunya infection, Dengue virus infection, viral encephalitis, Crimean-Congo hemorrhagic fever (CCHF) virus, Hendra virus infection, Nipah virus infection, and the West Nile virus infection [114]. Autophagosome formation is induced in the early stages of the influenza A virus. However, later stages result in the inhibition of autophagosomal maturation. MTORC1 downregulates classical swine fever virus replication through autophagy and IRES-dependent translation. Pathogenicity of the Dengue/Zika virus is controlled by antibody-dependent enhancement, which can cause autophagy induction in human umbilical vein endothelial cells. Transcription of ATGs is promoted in severe acute respiratory syndrome (SARS)-coronavirus. Moreover, it has been reported that endoplasmic reticulum (ER) stress in Dengue virus (DENV) infections led to autophagy activation, viral replication, and pathogenesis. Autophagy has also been shown to have a crucial role in microbial infections, including those caused by Listeria, Salmonella, Shigella, Streptococcus, Mycobacterium tuberculosis, and Salmonella enterica serovar Typhimurium [115]. The role of autophagy in Toxoplasmacidal mechanisms has been observed [116].

\section{Hematological and solid malignancies}

Autophagy is more involved in cancers than other diseases; the effects of autophagy on cancer are highly 
dependent on the situation. It can eliminate the tumor or be ineffective or even strengthen the spread of the tumor. In recent years many studies have been conducted to investigate the use of autophagy to treat diseases. However, the critical question of whether autophagy could be regulated for cancer treatment or not remained unanswered, although there are now pieces of evidence that autophagy in cancer acts as a lifeline to chemotherapy. Although, studies have shown that autophagy is a vital cell rescue mechanism for clearing defective proteins and damaged organelles that conserve cell energy and function $[21,117,118]$.

Plumbagin, a quinonoid component isolated from Plumbago zeylanica L., saikosaponin-d (Ssd) a triterpenoid saponin, berberine, carnosol, bufalin, licarin A isolated from Myristica fragrans, and isoliquiritigenin a flavonoid of Glycyrrhiza uralensis are examples of valuable phytochemicals which can induce cytotoxic autophagy in cancer cells [119].

The roles of miRNAs in osteosarcoma in recent investigations showed miRNAs like $M i R-22$ involved in determinant sectors of autophagy gene regulation. Beclin1, LC3, Metadherin (MTDH), ATG5 genes, and the proteins of $L C 3, A T G 5$ that are closely related to autophagy regulation and anticancer drug resistance, when an encounter with miR-22 downregulate obviously [120-122].

Lovastatin inhibition of autophagy in glioblastoma cells which were resistant to temozolomide, enhanced efficacy of temozolomide through cessation of autophagy cascade by inhibition of $L A M P 2$ and dynein proteins. Impermanent formation of autolysosomes increased glioblastoma cell death $[123,124]$.

Proof of bufalin-induced autophagy comprised the formation of the acidic vesicular organelles, augmentation of autophagolysosomes, and accumulation of LC3-II. More reports presented that the mechanism of bufalin-induced autophagy connected with ATP depletion involved an increased AMPK activation, diminished phosphorylation of mTOR, and its downstream targets $4 E B P 1$ and p70S6K1 [125].

Autophagy inhibition in lung cancer can overcome drug resistance, like the effect of candesartan and gingerol on TRAIL-resistant lung cancers via autophagy inhibition caused a reduction of drug resistance [126, 127].

A study on the effect of cigarette smoke extract (CSE) on fibroblast cells of the lung seen CSE induction of autophagy and related proteins, such as optineurin. Fibroblasts in the following showed a rise in the amount of IL-8, which caused elevation of cell malignancy potential. Inhibition optineurin reversed the pathway and reduced p62 protein and IL-8 expression in these treated fibroblasts. Thus, it seems that the IL-8 expression of cigarette smoke triggered autophagy can support the spread of cancerous lung cells [128].

Zhang and colleagues explored the linkage of autophagy to the effectiveness of chemotherapy with a regimen of 5-FU and 3-MA in the treatment of Squamous cell carcinoma (SCC). SCC is a type of malignant skin cancer, and its prevalence is one-fifth of non-melanoma skin cancers. Thus, the treatment and prevention of SCC recurrence are among dermatologists' and radiotherapists' fields [129].

3-Methyladenine (3-MA) is an inhibitor of autophagy by preventing the formation of autophagosomes and increases the sensitivity of SCC to radiotherapy, and increase the antitumor effect of 5-Fluorouracil (5-FU); in another study by Zhang and colleagues, they combined 5-aminolevulinic acid-photodynamic therapy (ALAPDT) with 5-FU and found that this treatment regimen with or without 5-FU or 3-MA Stimulates apoptosis, primarily if used with 5-FU and 3-MA, this treatment regimen on cell lines of A431 and A375, the repressed proliferation of cells and ALA-PDT induced apoptosis and this effect increased by 3-MA pretreatment, which seems autophagy regulation is involved in ALA-PDT action [130].

Yi Zhang and colleagues explored the effect of sodium selenite on leukemia. They found p53 was a primary up regulator of phospholipid scramblase 1 (PLSCR1), acting as a confluence shifting autophagy to apoptosis in leukemia. PLSCR1 Tuning of apoptosis and autophagy possibly is the primary mechanism in the efficacy of chemotherapy [76, 131]. Ectopic expression of Beclin1 in breast cancer cells (MCF-7) activated autophagy. Moreover, the monoallelic deletion of Beclin1 was witnessed in numerous specimens of human breast, ovarian, and prostate cancer cells [132].

UVRAG, an upregulating agent of the Beclin1-class III PI3K complex, suppressed the proliferation and tumorigenicity of colon cancer cells. In addition, knockout of Bif-1which interacted with Beclin1 and activated the Beclin1-class III PI3K complex, ameliorating spontaneous tumors' progression in vivo [132-134]. Zhao and coworkers indicated that miR-375/sorafenib in lipid-coated calcium carbonate nanoparticles might play a significant role in the therapeutic approach of hepatocellular cancer via inhibition of autophagy [63]. A systemic mosaic deletion of $A T G 5$ or a liver-specific ATG7 deficiency in mice led to the development of benign liver tumors, proposing the critical role of autophagy in the abrogation of spontaneous tumor genesis [135]. 
Aging, inflammatory diseases, and immune dysfunction Autophagy decreases during the aging process. It seems that maintaining autophagy is an effective anti-aging treatment. During the aging process, the innate immune system response to external antigen decreases, ROS production and IL-1B concentration, and IL-18 Increase by macrophages. Other innate immune cells that are affected by aging are neutrophils. Although there is no reduction in the total number of these cells during aging, studies have shown that their capacity for phagocytosis and xenophagy have an inverse relationship with age. They find similar conditions diminish antigens presentation and chemotaxis. In addition to the weakening of immunity against pathogens, chronic inflammation develops, and immune cells do not return to the basal level of homeostasis after inflammation. Peripheral cells such as adipose increase inflammatory status by increasing the release of cytokines. Cell-mediated immunity because of DNA damages and shortening of telomeres have obstacles to replicating and reproducing. Their ability to create a memory is reduced $[136,137]$.

$\mathrm{T}$ cells secrete proinflammatory cytokines without antigen stimulation, do not respond to apoptotic signals, diminished the production of the central cytokines like TNF- $\alpha$, IL-2, INF- $\gamma$, and granzyme B, and consequently, their response to infections decreases. Although $\mathrm{B}$ lymphocytes are less studied in aging research, in some studies on human vaccination, due to reduced humoral immune response, it appears to be related to B cells malfunction $[76,138]$. Furthermore, macrophages, $\mathrm{B}$, and $\mathrm{T}$ cells for phagocytosis, antigen presentation, and hemostasis require autophagy. Therefore, it is conceivable to reduce the immunological effects of aging by targeting autophagy [139].

Recent studies indicated that AMPK activation inhibited cell-induced aging by boosting activation of autophagy, detention of mTORC1 process, and reducing oxidative stress. Hence, it seems to be an excellent strategy to enable AMPK to stop cell aging [140, 141].

$A T G 16 L$ and autophagy stimulatory immunity-related GTPase (IRGM) are two genes involved in autophagy regulation related to Crohn's disease. $A T G 2 B$ and autophagy have been regulated by miR-143 in another study of Crohn's disease [142]. Blocking the differentiation and function of Treg cells by the interleukin-21/mTOR axis via autophagy inhibition in systemic lupus erythematosus patients has been targeted. Moreover, the ATG5 gene has participated in the incidence and development of systemic lupus erythematosus as an autoimmune disease [143]. Additionally, autophagy and chronic inflammation involved cystic fibrosis, an incurable genetic condition initiated by mutations in the gene encoding the cystic fibrosis transmembrane conductance regulator.
Moreover, the modulation of autophagy is essential in other inflammatory diseases like Behcet's disease and celiac disease [144, 145].

\section{Pulmonary diseases}

Meng and colleagues claimed autophagy could mitigate pulmonary fibrosis by mediating the activation of the NOD-like receptor family pyrin domain containing 3 (NLRP3) inflammasome induced by angiotensin IImediated ROS via modulation of redox balance [146]. Moreover, asthma and COPD can be related to the role of autophagy [144].

Evidence indicated that autophagy plays a central role in tissue remodeling. The changed autophagy pathway in response to cellular stress in asthma and COPD resulted in activation and crosstalk between structural airway and immune cells. This further leads to autophagy impairment causing intracellular constituents degradation and secreting inflammation mediators, which cause lung airway remodeling [144].

\section{Ocular diseases}

Corneal oxidative stress upregulated LC3, Beclin 1, and ATG12 levels; however, P62 were downregulated. Hence, various signaling pathways, such as the mTOR, showed the interplay of ROS and autophagy in corneas [147]. Regulation of the expression of $L C 3 I I$ and $P 62$ regarding suppression of the senescence of lens epithelial cells by restoring autophagy flux after metformin administration was observed. More studies about the role of autophagy in glaucoma have been reported [144]. Autophagy can act as a cyto-protector or a cyto-killer in glaucoma. The dual role of autophagy in glaucoma progression is related to the glaucoma stage, autophagy stage, autophagy detection time, and even genomic changes [144].

\section{Cardiometabolic diseases}

Cardiometabolic disease comprises a wide range of cardiovascular diseases, diabetes, and obesity [148]. In vitro and in vivo results elucidated that hindering the expression of ATG7 had a defective role regarding insulin signaling and augmented ER stress. On the contrary, reinstatement of $A T G 7$ expression in the liver limited ER stress and improved insulin activity and systemic glucose tolerance in mice with obesity. Furthermore, autophagy has a crucial role in normal adipogenesis, and abridge of autophagy has anti-obesity and insulin-sensitizing properties [149, 150].

According to the shreds of evidence, impaired autophagy might contribute to insulin deficiency and hyperglycemia. Autophagy was considered a significant regulator in pancreatic $\beta$-cells, and ATG7 mutation exhibited damaged glucose tolerance. In an investigation 
on the effect of liraglutide on cardiac fibrosis caused by aortic banding in rats, results indicated that inhibition of $p 70 S 6 K$, a ribosomal protein $\mathrm{S} 6$ kinase, and mTOR signaling by liraglutide, showing a positive effect on reduction of heart dysfunction emanating from fibrosis and cardiomyocyte hypertrophy by increase autophagy [151]. ATG5 deficiency was reported to contribute to various cardiovascular diseases [126].

\section{Renal diseases}

Dysregulation of autophagy is one of the reasons responsible for the pathogenesis of acute kidney injury, or incomplete kidney repair after acute kidney injury and chronic kidney disease of diverse aetiologies, comprising diabetic kidney disease, focal segmental glomerulosclerosis, and polycystic kidney disease. Autophagy also plays a critical role in kidney aging. CMA, AMPK, sirtuins, and the mTOR pathway are considered the most feasible gates for regulating autophagy-related to renal disease $[11,144,152]$.

\section{Liver diseases}

Autophagy has an emerging primary function in maintaining the balance of liver metabolism and the modulation of its physiology. On the contrary, numerous documents have shown that autophagy may diseasedependently play a part in the pathogenesis of liver disorders, like Wilson's disease, $\alpha-1$ antitrypsin deficiency, hepatocellular carcinoma, cirrhosis, acute liver injury, chronic alcohol-associated liver disease, liver hepatitis, fibrosis, steatosis, and non-alcoholic fatty liver disease (NAFLD) [153]. Carbamazepine, rapamycin, and ursodeoxycholic acid are drugs with autophagy induction and assistive in combating various liver diseases [153]. There are a number of transcription factors that can assist in regulating hepatic functions related to autophagy. These transcription factors are encompassing CREB, Nrf2, PPAR $\alpha$, and TFEB. Ueno et al. demonstrated that the mTORC1 complex acts as a fundamental portion of the liver's amendment to metabolic disorders, containing nutrient starvation. Inactivation of mTORC1 causes TFEB activation and NCOR1 inactivation. Moreover, the integral functioning of Nrf2 and Parkin-mediated mitophagy have participated in the modulation of autophagy in liver disorders [144, 154-156].

\section{Bone diseases}

Autophagic catabolism is involved in controlling and managing the survival and working of osteoclasts, osteocytes, and osteoblasts. Hence it is essential for the conservation of skeletal homeostasis. Unusual autophagic action causes obviation of the balance of the boneremodeling manifesting as pathological conditions, comprising osteopetrosis and osteoporosis. Regulation of autophagy has eliminated therapeutic potential in the treatment and prevention of bone-related diseases. ATG5, ATG10, ATG16L1, and p62/SQSTM1 are significant targets that can be attributed to Paget's disease [144, 157, 158].

\section{Skeletal muscle disorders}

According to the pivotal character of skeletal muscles in metabolism control, maintaining the muscle homeostasis through making balance among catabolic and anabolic processes is of high emphasis. Hence, autophagy is a principle catabolic mechanism in the skeletal muscles which helps in the achievement of the mentioned aim. The significance of autophagy as a helpful target and recommend explaining associations between protein unfurling and mTOR-dependent or mTOR-independent hypertrophic reactions is likely to uncover particular helpful windows for treating muscle squandering clutters (muscle function loss or paralysis) [19, 144].

\section{Congenital developmental disease}

While the appropriate function of autophagy is vital for the exact function and CNS development, any single gene disorder related to autophagy signaling can be harmful. Some of the gene disorders of autophagy signaling are stated as EPG5-associated Vici syndrome, SQSTM1/ p62-associated childhood-onset neurodegeneration, SNX14-associated autosomal-recessive spinocerebellar ataxia 20,WDR45-related $\beta$-propeller protein-associated neurodegeneration, ATG5-associated autosomal-recessive ataxia syndrome, and numerous types of hereditary spastic paraplegias $[144,159,160]$.

\section{Conclusion}

In summary, regarding the emerging emphasis on cellular autophagy, a comprehensive literature overview was conducted to discuss the pharmacological aspects of autophagy, focusing on its interplay with oxidative stress in neurological disorders. Various human diseases have been contributed to alterations in autophagy entailing cancers, cardiometabolic diseases, renal and liver diseases, bone diseases, neurological disease, aging, and immune dysfunctions. This study claimed some of the principal interrelations of autophagy and oxidative stress in cerebral ischemia, Huntington's disease, Parkinson's disease, Alzheimer's disease, cerebral hypoperfusion, and spinal cord injury. This interplay assists in the foundation of variable targets for drug discovery.

\section{Acknowledgements}

The figures were drawn using illustrations adapted from the image bank of Servier Medical Art (Cellular Biology; https://smart.servier.com/). 


\section{Authors' contributions}

All authors contributed to the manuscript. Conceptualization, MT and SS; validation investigation, resources, data extraction, and writing, all authors; review and editing, MT and SS, All authors read and approved the final manuscript.

\section{Funding}

Not applicable.

\section{Availability of data and materials}

The datasets used and/or analyzed during the current study are available in the body of main text.

\section{Declarations}

\section{Ethics approval and consent to participate}

Not applicable.

\section{Consent for publication}

Not applicable.

\section{Competing interests}

The authors confirm that the present article content has no conflict of interest.

\section{Author details}

${ }^{1}$ Department of Pharmacognosy, School of Pharmacy, Shahid Beheshti University of Medical Sciences, Tehran, Iran. ${ }^{2}$ Department of Pharmaceutical Biotechnology, School of Pharmacy, Shahid Beheshti University of Medical Sciences, Tehran, Iran. ${ }^{3}$ Department of Chemistry and Biochemistry, University of Texas at Arlington, Arlington, TX 76019, USA. ${ }^{4}$ Viatris Pharmaceuticals Inc, 3300 Research Plaza, San Antonio, TX 78235, USA. ${ }^{5}$ Medical Toxicology and Drug Abuse Research Center (MTDRC), Birjand University of Medical Sciences, Birjand, Iran. ${ }^{6}$ Faculty of Pharmacy, Birjand University of Medical Sciences, Birjand, Iran. ${ }^{7}$ Noncommunicable Diseases Research Center, Neyshabur University of Medical Sciences, Neyshabur, Iran.

\section{Received: 11 May 2021 Accepted: 17 January 2022}

Published online: 29 January 2022

\section{References}

1. Wang M-M, Feng Y-S, Yang S-D, Xing Y, Zhang J, Dong F, et al. The relationship between autophagy and brain plasticity in neurological diseases. Front Cell Neurosci. 2019;13:228.

2. Farkhondeh T, Samarghandian S, Pourbagher-Shahri AM, Sedaghat M. The impact of curcumin and its modified formulations on Alzheimer's disease. J Cell Physiol. 2019;234(10):16953-65.

3. Klionsky DJ. Autophagy: from phenomenology to molecular understanding in less than a decade. Nat Rev Mol Cell Biol. 2007;8(11):931-7.

4. Wang S-Y, Yu Q-J, Zhang R-D, Liu B. Core signaling pathways of survival/ death in autophagy-related cancer networks. Int J Biochem Cell Biol. 2011;43(9):1263-6.

5. Zhang J. Autophagy and mitophagy in cellular damage control. Redox Biol. 2013;1(1):19-23.

6. Kaushik S, Rodriguez-Navarro JA, Arias E, Kiffin R, Sahu S, Schwartz GJ, et al. Autophagy in hypothalamic AgRP neurons regulates food intake and energy balance. Cell Metabol. 2011;14(2):173-83.

7. Rahman MA, Rahman MR, Zaman T, Uddin MS, Islam R, Abdel-Daim MM, et al. Emerging potential of naturally occurring autophagy modulators against neurodegeneration. Curr Pharm Des. 2020;26(7):772-9.

8. Kesidou E, Lagoudaki R, Touloumi O, Poulatsidou K-N, Simeonidou C. Autophagy and neurodegenerative disorders. Neural Regen Res. 2013;8(24):2275.

9. Batatinha HAP, Diniz TA, de Souza Teixeira AA, Krüger K, Rosa-Neto JC. Regulation of autophagy as a therapy for immunosenescence-driven cancer and neurodegenerative diseases: The role of exercise. J Cell Physiol. 2019;234(9):14883-95.

10. Mizushima N, Levine B, Cuervo AM, Klionsky DJ. Autophagy fights disease through cellular self-digestion. Nature. 2008;451(7182):1069-75.
11. Tang C, Livingston MJ, Liu Z, Dong Z. Autophagy in kidney homeostasis and disease. Nat Rev Nephrol. 2020;16(9):489-508.

12. Handy DE, Loscalzo J. Redox regulation of mitochondrial function. Antioxid Redox Signal. 2012;16(11):1323-67.

13. Yun HR, Jo YH, Kim J, Shin Y, Kim SS, Choi TG. Roles of autophagy in oxidative stress. Int J Mol Sci. 2020;21(9):3289.

14. Hajzadeh MA, Rajaei Z, Shafiee S, Alavinejhad A, Samarghandian S, Ahmadi M. Effect of barberry fruit (berberis vulgaris) o serum glucose ad lipids i streptozotoci-diabetic rats. Pharmacol Online. 2011;1:809-17.

15. Samarghandian S, Samini F, Azimi-Nezhad M, Farkhondeh T. Antioxidative effects of safranal on immobilization-induced oxidative damage in rat brain. Neurosci Lett. 2017 Oct 17;659:26-32. doi: 10.1016/j. neulet.2017.08.065

16. Zhu J, Dagda RK, Chu CT. Monitoring mitophagy in neuronal cell cultures. In: Manfredi G, Kawamata H, editors. Neurodegeneration. Cham: Springer; 2011. p. 325-39.

17. Singh SS, Vats S, Chia AY-Q, Tan TZ, Deng S, Ong MS, et al. Dual role of autophagy in hallmarks of cancer. Oncogene. 2018;37(9):1142-58.

18. Fujita N, Itoh T, Omori H, Fukuda M, Noda T, Yoshimori T. The Atg16L complex specifies the site of LC3 lipidation for membrane biogenesis in autophagy. Mol Biol Cell. 2008;19(5):2092-100.

19. Grumati P, Bonaldo P. Autophagy in skeletal muscle homeostasis and in muscular dystrophies. Cells. 2012;1(3):325-45.

20. Desideri E, Vegliante R, Cardaci S, Nepravishta R, Paci M, Ciriolo MR. MAPK14/p38a-dependent modulation of glucose metabolism affects ROS levels and autophagy during starvation. Autophagy. 2014:10(9):1652-65.

21. Du J, Liang Y, Xu F, Sun B, Wang Z. Trehalose rescues Alzheimer's disease phenotypes in APP/PS1 transgenic mice. J Pharm Pharmacol. 2013;65(12):1753-6

22. Ashrafizadeh M, Ahmadi Z, Farkhondeh T, Samarghandian S. Modulatory effects of statins on the autophagy: a therapeutic perspective. J Cell Physiol. 2020;235(4):3157-68.

23. Ashrafizadeh M, Tavakol S, Ahmadi Z, Roomiani S, Mohammadinejad R, Samarghandian S. Therapeutic effects of kaempferol affecting autophagy and endoplasmic reticulum stress. Phytother Res. 2020;34(5):911-23.

24. Ashrafizadeh M, Zarrabi A, Orouei S, Hushmandi K, Hakimi A, Zabolian A, et al. MicroRNA-mediated autophagy regulation in cancer therapy: the role in chemoresistance/chemosensitivity. Eur J Pharmacol. 2020. https://doi.org/10.1016/j.ejphar.2020.173660.

25. Desai S, Juncker M, Kim C. Regulation of mitophagy by the ubiquitin pathway in neurodegenerative diseases. Exp Biol Med. 2018;243(6):554-62.

26. Bhattacharyya A, Chattopadhyay R, Mitra S, Crowe SE. Oxidative stress: an essential factor in the pathogenesis of gastrointestinal mucosal diseases. Physiol Rev. 2014;94(2):329-54.

27. Talebi M, Mojab F. A systematic review of preclinical and clinical studies on therapeutic potential of piper nigrum on cognitive impairment in Alzheimer's disease and other biological conditions of memory loss. Int Pharm Acta. 2020. https://doi.org/10.3390/ijms20092313.

28. Talebi M, Il gün S, Ebrahimi V, Talebi M, Farkhondeh T, Ebrahimi H, et al. Zingiber officinale ameliorates Alzheimer's disease and cognitive impairments: lessons from preclinical studies. Biomed Pharmacother. 2021. https://doi.org/10.1016/j.biopha.2020.111088.

29. Talebi M, Kakouri E, Talebi M, Tarantilis PA, Farkhondeh T, Illgün S, et al. Nutraceuticals-based therapeutic approach: recent advances to combat pathogenesis of Alzheimer's disease. Expert Rev Neurother. 2021;21(6):625-42.

30. Bonomini F, Rodella LF, Rezzani R. Metabolic syndrome, aging and involvement of oxidative stress. Aging Dis. 2015;6(2):109-20.

31. Dewaele M, Maes H, Agostinis P. ROS-mediated mechanisms of autophagy stimulation and their relevance in cancer therapy. Autophagy. 2010:6(7):838-54.

32. Fransen $M$, Lismont $C$. Redox signaling from and to peroxisomes: progress, challenges, and prospects. Antioxid Redox Signal. 2019;30(1):95-112.

33. Talebi M, Talebi M, Kakouri E, Farkhondeh T, Pourbagher-Shahri AM, Tarantilis PA, et al. Tantalizing role of p53 molecular pathways and its coherent medications in neurodegenerative diseases. Int J Biol Macromol. 2021;172:93-103 
34. Lan A-P, Chen J, Zhao Y, Chai Z, Hu Y. mTOR signaling in Parkinson's disease. Neuromolecular Med. 2017;19(1):1-10.

35. Lipton JO, Sahin M. The neurology of mTOR. Neuron. 2014;84(2):275-91.

36. Alexander A, Cai S-L, Kim J, Nanez A, Sahin M, MacLean KH, et al. ATM signals to TSC2 in the cytoplasm to regulate mTORC1 in response to ROS. Proc Natl Acad Sci. 2010;107(9):4153-8.

37. Kongara $S$, Karantza $V$. The interplay between autophagy and ROS in tumorigenesis. Front Oncol. 2012;2:171.

38. Azad MB, Chen Y, Gibson SB. Regulation of autophagy by reactive oxygen species (ROS): implications for cancer progression and treatment. Antioxid Redox Signal. 2009;11(4):777-90.

39. Samarghandian S, Azimi-Nezhad M, Farkhondeh T. Thymoquinoneinduced antitumor and apoptosis in human lung adenocarcinoma cells. J Cell Physiol. 2019;234(7):10421-31.

40. Talebi M, Talebi M, Farkhondeh T, Mishra G, Illgün S, Samarghandian S. New insights into the role of the Nrf2 signaling pathway in green tea catechin applications. Phytother Res. 2021;35(6):3078-112.

41. Pajares M, Jiménez-Moreno N, García-Yagüe ÁJ, Escoll M, de Ceballos ML, Van Leuven F, et al. Transcription factor NFE2L2/NRF2 is a regulator of macroautophagy genes. Autophagy. 2016;12(10):1902-16.

42. Zhao RZ, Jiang S, Zhang L, Yu ZB. Mitochondrial electron transport chain, ROS generation and uncoupling. Int J Mol Med. 2019;44(1):3-15.

43. Dong X-X, Wang Y, Qin Z-H. Molecular mechanisms of excitotoxicity and their relevance to pathogenesis of neurodegenerative diseases. Acta Pharmacol Sin. 2009;30(4):379-87.

44. Funderburk SF, Marcellino BK, Yue Z. Cell, "self-eating"(autophagy) mechanism in Alzheimer's disease. Mt Sinai J Med. 2010;77(1):59-68.

45. Essick EE, Sam F. Oxidative stress and autophagy in cardiac disease, neurological disorders, aging and cancer. Oxid Med Cell Longev. 2010;3:593214.

46. Cheng W-T, Guo Z-X, Lin C-A, Lin M-Y, Tung L-C, Fang K. Oxidative stress promotes autophagic cell death in human neuroblastoma cells with ectopic transfer of mitochondrial PPP2R2B (Bbeta2). BMC Cell Biol. 2009. https://doi.org/10.1186/1471-2121-10-91.

47. Choi KC, Kim SH, Ha JY, Kim ST, Son JH. A novel mTOR activating protein protects dopamine neurons against oxidative stress by repressing autophagy related cell death. J Neurochem. 2010;112(2):366-76.

48. Talebi M, Talebi M, Samarghandian S. Association of Crocus sativus with cognitive dysfunctions and Alzheimer's disease: a systematic review. Biointerface Res Appl Chem. 2021;11(1):7468-92.

49. Uddin MS, Kabir MT, Tewari D, Mamun AA, Mathew B, Aleya L, et al. Revisiting the role of brain and peripheral $A \beta$ in the pathogenesis of Alzheimer's disease. J Neurol Sci. 2020;416:116974.

50. Talebi M, Esmaeeli H, Talebi M, Farkhondeh T, Samarghandian S. A Concise Overview of Biosensing Technologies for the Detection of Alzheimer's Disease Biomarkers. Curr Pharm Biotechnol. 2021. https:// doi.org/10.2174/2666796702666210709122407.

51. Duncan RS, Song B, Koulen P. Presenilins as drug targets for Alzheimer's disease-recent insights from cell biology and electrophysiology as novel opportunities in drug development. Int J Mol Sci. 2018;19(6):1621.

52. Uddin M, Stachowiak A, Mamun AA, Tzvetkov NT, Takeda S, Atanasov AG, et al. Autophagy and Alzheimer's disease: from molecular mechanisms to therapeutic implications. Front Aging Neurosci. 2018;10:4.

53. Gorantla NV, Chinnathambi S. Autophagic pathways to clear the tau aggregates in Alzheimer's disease. Cell Mol Neurobiol. 2020;6:1-7.

54. Di Domenico F, Barone E, Perluigi M, Butterfield DA. The triangle of death in Alzheimer's disease brain: the aberrant cross-talk among energy metabolism, mammalian target of rapamycin signaling, and protein homeostasis revealed by redox proteomics. Antioxid Redox Signal. 2017;26(8):364-87.

55. Huynh KK, Eskelinen EL, Scott CC, Malevanets A, Saftig P, Grinstein S. LAMP proteins are required for fusion of lysosomes with phagosomes. EMBO J. 2007;26(2):313-24.

56. Uddin MS, Rahman MA, Kabir MT, Behl T, Mathew B, Perveen A, et al. Multifarious roles of mTOR signaling in cognitive aging and cerebrovascular dysfunction of Alzheimer's disease. IUBMB Life. 2020;72(9):1843-55

57. Huang J-L, Su M, Wu D-P. Functional roles of circular RNAs in Alzheimer's disease. Ageing Res Rev. 2020;60:101058.
58. Kim J, Yoon H, Kim J. Role of Autophagy in Alzheimer's Disease. Curr Enzym Inhib. 2013;9(1):55-66.

59. Du F, Yu Q, Yan S, Hu G, Lue L-F, Walker DG, et al. PINK1 signalling rescues amyloid pathology and mitochondrial dysfunction in Alzheimer's disease. Brain. 2017;140(12):3233-51.

60. Li L. The molecular mechanism of glucagon-like peptide-1 therapy in Alzheimer's disease, based on a mechanistic target of rapamycin pathway. CNS Drugs. 2017;31(7):535-49.

61. Zheng X, Wang W, Liu R, Huang H, Zhang R, Sun L. Effect of p62 on tau hyperphosphorylation in a rat model of Alzheimer's disease. Neural Regen Res. 2012;7(17):1304.

62. Agrawal I, Jha S. Mitochondrial dysfunction and Alzheimer's disease: Role of microglia. Front Aging Neurosci. 2020;12:252.

63. Zhang Y-d, Zhao J-j. TFEB participates in the A $\beta$-induced pathogenesis of Alzheimer's disease by regulating the autophagy-lysosome pathway. DNA Cell Biol. 2015;34(11):661-8.

64. Omata Y, Lim Y-M, Akao Y, Tsuda L. Age-induced reduction of autophagy-related gene expression is associated with onset of Alzheimer's disease. Am J Neurodegener Dis. 2014;3(3):134.

65. Li L-H, Peng W-N, Deng Y, Li J-J, Tian X-R. Action of trichostatin A on Alzheimer's disease-like pathological changes in SH-SY5Y neuroblastoma cells. Neural Regen Res. 2020;15(2):293.

66. Moon J-H, Jeong J-K, Hong J-M, Seol J-W, Park S-Y. Inhibition of autophagy by captopril attenuates prion peptide-mediated neuronal apoptosis via AMPK activation. Mol Neurobiol. 2019;56(6):4192-202.

67. Cai Z, Yan L-J, Li K, Quazi SH, Zhao B. Roles of AMP-activated protein kinase in Alzheimer's disease. Neuromolecular Med. 2012;14(1):1-14.

68. Weng M-H, Chen S-Y, Li Z-Y, Yen G-C. Camellia oil alleviates the progression of Alzheimer's disease in aluminum chloride-treated rats. Free Radic Biol Med. 2020;152:411-21.

69. Anglade P, Vyas S, Javoy-Agid F, Herrero MT, Michel PP, Marquez J, et al. Apoptosis and autophagy in nigral neurons of patients with Parkinson's disease. Histol Histopathol. 1997;12(1):25-31.

70. Ludtmann MHR, Abramov AY. Mitochondrial calcium imbalance in Parkinson's disease. Neurosci Lett. 2018:663:86-90.

71. Jankovic J, Tan EK. Parkinson's disease: etiopathogenesis and treatment. J Neurol Neurosurg Psychiatry Res. 2020;91(8):795-808.

72. Inamdar NN, Arulmozhi DK, Tandon A, Bodhankar SL. Parkinson's disease: genetics and beyond. Curr Neuropharmacol. 2007;5(2):99-113.

73. Dagda RK, Zhu J, Chu CT. Mitochondrial kinases in Parkinson's disease: converging insights from neurotoxin and genetic models. Mitochondrion. 2009;9(5):289-98.

74. Lu J, Wu M, Yue Z. Autophagy and Parkinson's disease. Autophagy: biology and diseases. Cham: Springer; 2020. p. 21-51.

75. Butler D, Bahr BA. Oxidative stress and lysosomes: CNS-related consequences and implications for lysosomal enhancement strategies and induction of autophagy. Antioxid Redox Signal. 2006;8(1-2):185-96.

76. Chatta GS, Price TH, Stratton JR, Dale DC. Aging and marrow neutrophil reserves. J Am Geriatr Soc. 1994:42(1):77-81.

77. Zhuang X-X, Wang S-F, Tan Y, Song J-X, Zhu Z, Wang Z-Y, et al. Pharmacological enhancement of TFEB-mediated autophagy alleviated neuronal death in oxidative stress-induced Parkinson's disease models. Cell Death Dis. 2020;11(2):1-18.

78. Ning B, Zhang Q, Wang N, Deng M, Fang Y. $\beta$-Asarone regulates ER stress and autophagy via inhibition of the PERK/CHOP/BCl-2/Beclin-1 Pathway in 6-OHDA-induced parkinsonian rats. Neurochem Res. 2019:44(5):1159-66.

79. Oh SE, Mouradian MM. Regulation of signal transduction by DJ-1. DJ-1/ PARK7 Protein. Cham: Springer; 2017. p. 97-131.

80. Ebert AD, Beres AJ, Barber AE, Svendsen CN. Human neural progenitor cells over-expressing IGF-1 protect dopamine neurons and restore function in a rat model of Parkinson's disease. Exp Neurol. 2008;209(1):213-23

81. Wang X-W, Yuan L-J, Yang Y, Zhang M, Chen W-F. IGF-1 inhibits MPTP/ MPP+-induced autophagy on dopaminergic neurons through the IGF1R/PI3K-Akt-mTOR pathway and GPER. Am J Physiol Endocrinol Metab. 2020;319(4):E734-43.

82. Zhu J, Gao W, Shan X, Wang C, Wang H, Shao Z, et al. Apelin-36 mediates neuroprotective effects by regulating oxidative stress, autophagy and apoptosis in MPTP-induced Parkinson's disease model mice. Brain Res. 2020;1726:146493. 
83. Zhang L, Zhang L, Li L, Hölscher C. Neuroprotective effects of the novel GLP-1 long acting analogue semaglutide in the MPTP Parkinson's disease mouse model. Neuropeptides. 2018;71:70-80.

84. Mao K, Chen J, Yu H, Li H, Ren Y, Wu X, et al. Poly (ADP-ribose) polymerase 1 inhibition prevents neurodegeneration and promotes $a$-synuclein degradation via transcription factor EB-dependent autophagy in mutant a-synucleinA53T model of Parkinson's disease. Aging Cell. 2020;19(6):e13163.

85. Lin C-H, Wei P-C, Chen C-M, Huang Y-T, Lin J-L, Lo Y-S, et al. Lactulose and melibiose attenuate MPTP-induced Parkinson's disease in mice by inhibition of oxidative stress, reduction of neuroinflammation and upregulation of autophagy. Front Aging Neurosci. 2020. https://doi.org/ 10.3389/fnagi.2020.00226.

86. Guo Y-L, Duan W-J, Lu D-H, Ma X-H, Li X-X, Li Z, et al. Autophagydependent removal of a-synuclein: a novel mechanism of GM1 ganglioside neuroprotection against Parkinson's disease. Acta Pharmacol Sin. 2021;42(4):518-28.

87. $\mathrm{He} X-\mathrm{H}$, Lin $\mathrm{F}$, Qin Z-H. Current understanding on the pathogenesis of polyglutamine diseases. Neurosci Bull. 2010;26(3):247-56.

88. Tsunemi T, Ashe TD, Morrison BE, Soriano KR, Au J, Roque RAV, et al. PGC-1 a rescues Huntington's disease proteotoxicity by preventing oxidative stress and promoting TFEB function. Sci Transl Med. 2012;4(142):142ra97.

89. Cortes CJ, La Spada AR. The many faces of autophagy dysfunction in Huntington's disease: from mechanism to therapy. Drug Discov Today. 2014;19(7):963-71.

90. An T, Shi P, Duan W, Zhang S, Yuan P, Li Z, et al. Oxidative stress and autophagic alteration in brainstem of SOD1-G93A mouse model of ALS. Mol Neurobiol. 2014;49(3):1435-48.

91. Deng Z, Sheehan P, Chen S, Yue Z. Is amyotrophic lateral sclerosis/ frontotemporal dementia an autophagy disease? Mol Neurodegener. 2017;12(1):1-11.

92. Ma L, Herren AW, Espinal G, Randol J, McLaughlin B, MartinezCerdeño V, et al. Composition of the intranuclear inclusions of fragile $X$-associated tremor/ataxia syndrome. Acta Neuropathol Commun. 2019;7(1):1-26

93. Wardman JH, Henriksen EE, Marthaler AG, Nielsen JE, Nielsen TT. Enhancement of autophagy and solubilization of ataxin-2 alleviate apoptosis in spinocerebellar ataxia type 2 patient cells. Cerebellum. 2020;19(2):165-81.

94. Morani F, Doccini S, Sirica R, Paterno M, Pezzini F, Ricca I, et al. Functional transcriptome analysis in ARSACS KO cell model reveals a role of sacsin in autophagy. Sci Rep. 2019;9(1):1-16.

95. Ye B, Wang Q, Hu H, Shen Y, Fan C, Chen P, et al. Restoring autophagic flux attenuates cochlear spiral ganglion neuron degeneration by promoting TFEB nuclear translocation via inhibiting MTOR. Autophagy. 2019;15(6):998-1016.

96. Lin L, Yee SW, Kim RB, Giacomini KM. SLC transporters as therapeutic targets: emerging opportunities. Nat Rev Drug Discov. 2015;14(8):543-60.

97. Yamada D, Kawabe K, Tosa I, Tsukamoto S, Nakazato R, Kou M, et al. Inhibition of the glutamine transporter SNAT1 confers neuroprotection in mice by modulating the mTOR-autophagy system. Commun Biol. 2019:2(1):1-11.

98. Mackenzie B, Schäfer MKH, Erickson JD, Hediger MA, Weihe E, Varoqui $H$. Functional properties and cellular distribution of the system A glutamine transporter SNAT1 support specialized roles in central neurons. J Biol Chem. 2003;278(26):23720-30.

99. Hägglund MGA, Sreedharan S, Nilsson VCO, Shaik JHA, Almkvist IM, Bäcklin S, et al. Identification of SLC38A7 (SNAT7) protein as a glutamine transporter expressed in neurons. J Biol Chem. 2011;286(23):20500-11.

100. Bagchi S, Baomar HA, Al-Walai S, Al-Sadi S, Fredriksson R. Histological analysis of SLC38A6 (SNAT6) expression in mouse brain shows selective expression in excitatory neurons with high expression in the synapses. PLoS ONE. 2014;9(4):e95438.

101. Papadakis M, Hadley G, Xilouri M, Hoyte LC, Nagel S, McMenamin MM, et al. Tsc1 (hamartin) confers neuroprotection against ischemia by inducing autophagy. Nat Med. 2013;19(3):351-7.

102. Liu Y, Xue X, Zhang H, Che X, Luo J, Wang P, et al. Neuronal-targeted TFEB rescues dysfunction of the autophagy-lysosomal pathway and alleviates ischemic injury in permanent cerebral ischemia. Autophagy. 2019;15(3):493-509.

103. Aredia F, Scovassi Al. A new function for miRNAs as regulators of autophagy. Future Med Chem. 2017;9(1):25-36.

104. Wang Y, Meng C, Zhang J, Wu J, Zhao J. Inhibition of GSK-3 $\beta$ alleviates cerebral ischemia/reperfusion injury in rats by suppressing NLRP3 inflammasome activation through autophagy. Int Immunopharmacol. 2019;68:234-41.

105. Fang C, Gu L, Smerin D, Mao S, Xiong X. The interrelation between reactive oxygen species and autophagy in neurological disorders. Oxid Med Cell Longev. 2017. https://doi.org/10.1155/2017/8495160.

106. Liu S, Su Y, Sun B, Hao R, Pan S, Gao X, et al. Luteolin protects against CIRI, potentially via regulation of the SIRT3/AMPK/mTOR signaling pathway. Neurochem Res. 2020;45(10):2499-515.

107. Su SH, Wang YQ, Wu YF, Wang DP, Lin Q, Hai J. Cannabinoid receptor agonist WIN55,212-2 and fatty acid amide hydrolase inhibitor URB597 may protect against cognitive impairment in rats of chronic cerebral hypoperfusion via PI3K/AKT signaling. Behav Brain Res. 2016;313:334-44

108. Su SH, Wu YF, Lin Q, Yu F, Hai J. Cannabinoid receptor agonist WIN55,212-2 and fatty acid amide hydrolase inhibitor URB597 suppress chronic cerebral hypoperfusion-induced neuronal apoptosis by inhibiting c-Jun $\mathrm{N}$-terminal kinase signaling. Neuroscience. 2015;301:563-75.

109. Su SH, Wu YF, Lin Q, Hai J. Cannabinoid receptor agonist WIN55,212-2 and fatty acid amide hydrolase inhibitor URB597 ameliorate neuroinflammatory responses in chronic cerebral hypoperfusion model by blocking NF-kB pathways. Naunyn Schmiedebergs Arch Pharmacol. 2017;390(12):1189-200.

110. Wang D, Lin Q, Su S, Liu K, Wu Y, Hai J. URB597 improves cognitive impairment induced by chronic cerebral hypoperfusion by inhibiting mTOR-dependent autophagy. Neuroscience. 2017;344:293-304.

111. Zhang D, Zhu D, Wang F, Zhu J-C, Zhai X, Yuan Y, et al. Therapeutic effect of regulating autophagy in spinal cord injury: a network meta-analysis of direct and indirect comparisons. Neural Regen Res. 2020;15(6):1120-32

112. Zhu Y, Tang Q, Wang G, Han R. Tanshinone IIA protects hippocampal neuronal cells from reactive oxygen species through changes in autophagy and activation of phosphatidylinositol 3-kinase, protein kinas $B$, and mechanistic target of rapamycin pathways. Curr Neurovasc Res. 2017;14(2):132-40.

113. Saha S, Panigrahi DP, Patil S, Bhutia SK. Autophagy in health and disease: a comprehensive review. Biomed Pharmacother. 2018;104:485-95.

114. Brien JD, Uhrlaub JL, Hirsch A, Wiley CA, Nikolich-Žugich J. Key role of T cell defects in age-related vulnerability to West Nile virusT cell defects and age-related vulnerability to WNV. J Exp Med. 2009;206(12):2735-45

115. Khandia R, Dadar M, Munjal A, Dhama K, Karthik K, Tiwari R, et al. A comprehensive review of autophagy and its various roles in infectious, noninfectious, and lifestyle diseases: current knowledge and prospects for disease prevention, novel drug design, and therapy. Cells. 2019;8(7):674

116. Krishnamurthy S, Konstantinou EK, Young LH, Gold DA, Saeij JPJ. The human immune response to Toxoplasma: autophagy versus cell death. PLoS Pathog. 2017:13(3):e1006176.

117. Rebecca WW, Amaravadi RK. Emerging strategies to effectively target autophagy in cancer. Oncogene. 2016;35(1):1-11.

118. Jiang G-M, Tan Y, Wang H, Peng L, Chen H-T, Meng X-J, et al. The relationship between autophagy and the immune system and its applications for tumor immunotherapy. Mol Cancer. 2019;18(1):1-22.

119. Sun $C-Y$, Zhang Q-Y, Zheng G-J, Feng B. Autophagy and its potent modulators from phytochemicals in cancer treatment. Cancer Chemother Pharmacol. 2019;83(1):17-26.

120. Camuzard O, Santucci-Darmanin S, Carle GF, Pierrefite-Carle V. Role of autophagy in osteosarcoma. J Bone Oncol. 2019;16:100235.

121. Wang P, Zhao ZQ, Guo SB, Yang TY, Chang ZQ, Li DH, et al. Roles of microRNA-22 in suppressing proliferation and promoting sensitivity of osteosarcoma cells via metadherin-mediated autophagy. Orthop Surg. 2019;11(2):285-93.

122. Jamali Z, Taheri-Anganeh M, Shabaninejad Z, Keshavarzi A, Taghizadeh $\mathrm{H}$, Razavi ZS, et al. Autophagy regulation by microRNAs: Novel insights into osteosarcoma therapy. IUBMB Life. 2020;72(7):1306-21. 
123. Zhu Z, Zhang P, Li N, Kiang KMY, Cheng SY, Wong VKW, et al. Lovastatin enhances cytotoxicity of temozolomide via impairing autophagic flux in glioblastoma cells. Biomed Res Int. 2019. https://doi.org/10.1155/ 2019/2710693.

124. Mora R, Régnier-Vigouroux A. Autophagy-driven cell fate decision maker: activated microglia induce specific death of glioma cells by a blockade of basal autophagic flux and secondary apoptosis/necrosis. Autophagy. 2009;5(3):419-21.

125. Shen $S$, Zhang $Y$, Wang $Z$, Zhang $R$, Gong $X$. Bufalin induces the interplay between apoptosis and autophagy in glioma cells through endoplasmic reticulum stress. Int J Biol Sci. 2014;10(2):212.

126. Nazim UM, Jeong J-K, Seol J-W, Hur J, Eo S-K, Lee J-H, et al. Inhibition of the autophagy flux by gingerol enhances TRAIL-induced tumor cell death. Oncol Rep. 2015;33(5):2331-6.

127. Rasheduzzaman M, Park S-Y. Antihypertensive drug-candesartan attenuates TRAIL resistance in human lung cancer via AMPK-mediated inhibition of autophagy flux. Exp Cell Res. 2018;368(1):126-35.

128. Hou HH, Pan HJ, Liao WY, Lee CH, Yu CJ. Autophagy in fibroblasts induced by cigarette smoke extract promotes invasion in lung cancer cells. Int J Cancer. 2020;147(9):2587-96.

129. Zhang L, Zhang J, Chen L, Wang J. Autophagy in human skin squamous cell carcinoma: Inhibition by 3-MA enhances the effect of 5-FU-induced chemotherapy sensitivity. Oncol Rep. 2015;34(6):3147-55.

130. Zhang L, Ji Z, Zhang J, Yang S. Photodynamic therapy enhances skin cancer chemotherapy effects through autophagy regulation. Photodiagnosis Photodyn Ther. 2019;28:159-65.

131. Zhang Y, Du Y, Le W, Wang K, Kieffer N, Zhang J. Redox control of the survival of healthy and diseased cells. Antioxid Redox Signal. 2011;15(11):2867-908

132. Mancias JD, Kimmelman AC. Mechanisms of selective autophagy in normal physiology and cancer. J Mol Biol. 2016:428(9):1659-80.

133. Jiang P, Mizushima N. Autophagy and human diseases. Cell Res. 2014;24(1):69-79.

134. Takahashi Y, Coppola D, Matsushita N, Cualing HD, Sun M, Sato Y, et al. Bif-1 interacts with Beclin 1 through UVRAG and regulates autophagy and tumorigenesis. Nat Cell Biol. 2007;9(10):1142-51.

135. Yun CW, Lee SH. The roles of autophagy in cancer. Int J Mol Sci. 2018;19(11):3466.

136. Grolleau-Julius A, Harning EK, Abernathy LM, Yung RL. Impaired dendritic cell function in aging leads to defective antitumor immunity. Cancer Res. 2008;68(15):6341-9.

137. Hazeldine J, Lord JM, Hampson P. Immunesenescence and inflammaging: a contributory factor in the poor outcome of the geriatric trauma patient. Ageing Res Rev. 2015;24:349-57.

138. Solana R, Pawelec G, Tarazona R. Aging and Innate Immunity. Immunity 2006;24(5):491-4

139. Pulko V, Davies JS, Martinez C, Lanteri MC, Busch MP, Diamond MS, et al. Human memory $T$ cells with a naive phenotype accumulate with aging and respond to persistent viruses. Nat Immunol. 2016;17(8):966-75.

140. Lin S-C, Hardie DG. AMPK: sensing glucose as well as cellular energy status. Cell Metabol. 2018;27(2):299-313.

141. Pourbagher-Shahri AM, Farkhondeh T, Talebi M, Kopustinskiene DM, Samarghandian S, Bernatoniene J. An overview of NO signaling pathways in aging. Molecules. 2021. https://doi.org/10.3390/molecules2 6154533

142. Lin X-T, Zheng X-B, Fan D-J, Yao Q-Q, Hu J-C, Lian L, et al. MicroRNA-143 targets ATG2B to inhibit autophagy and increase inflammatory responses in Crohn's disease. Inflamm Bowel Dis. 2018;24(4):781-91.

143. Kato H, Perl A. Blockade of treg cell differentiation and function by the interleukin-21-mechanistic target of rapamycin axis via suppression of autophagy in patients with systemic lupus erythematosus. Arthritis Rheumatol. 2018;70(3):427-38.

144. Levine $B$, Kroemer $G$. Biological functions of autophagy genes: a disease perspective. Cell. 2019:176(1):11-42.

145. Xie W, Zhou J. Aberrant regulation of autophagy in mammalian diseases. Biol Lett. 2018;14(1):20170540.

146. Meng Y, Pan M, Zheng B, Chen Y, Li W, Yang Q, et al. Autophagy attenuates angiotensin II-induced pulmonary fibrosis by inhibiting redox imbalance-mediated NOD-like receptor family pyrin domain containing 3 inflammasome activation. Antioxid Redox Signal. 2019;30(4):520-41.
147. Yin Y, Zong R, Bao X, Zheng X, Cui H, Liu Z, et al. Oxidative stress suppresses cellular autophagy in corneal epithelium. Invest Ophthalmol Vis Sci. 2018:59(8):3286-93.

148. Farkhondeh T, Samarghandian S, Azimi-Nezhad M. The role of arsenic in obesity and diabetes. J Cell Physiol. 2019;234(8):12516-29.

149. Samarghandian S, Samini F, Azimi-Nezhad M, Farkhondeh T. Anti-oxidative effects of safranal on immobilization-induced oxidative damage in rat brain. Neurosci Lett. 2017;659:26-32.

150. Qian Q, Zhang Z, Orwig A, Chen S, Ding W-X, Xu Y, et al. S-Nitrosoglutathione Reductase Dysfunction Contributes to Obesity-Associated Hepatic Insulin Resistance via Regulating Autophagy. Diabetes. 2018:67(2):193.

151. Zheng R-H, Zhang W-W, Ji Y-N, Bai X-J, Yan C-P, Wang J, et al. Exogenous supplement of glucagon like peptide-1 protects the heart against aortic banding induced myocardial fibrosis and dysfunction through inhibiting mTOR/p70S6K signaling and promoting autophagy. Eur J Pharmacol. 2020;883:173318.

152. Lenoir O, Tharaux P-L, Huber TB. Autophagy in kidney disease and aging: lessons from rodent models. Kidney Int. 2016;90(5):950-64.

153. Allaire M, Rautou P-E, Codogno P, Lotersztajn S. Autophagy in liver diseases: time for translation? J Hepatol. 2019;70(5):985-98.

154. Ueno T, Komatsu M. Autophagy in the liver: functions in health and disease. Nat Rev Gastroenterol Hepatol. 2017;14(3):170-84.

155. Yazdani E, Talebi M, Zarshenas M, Moein M. Evaluation of possible antioxidant activities of barberry solid formulation, a selected formulation from Traditional Persian Medicine (TPM) via various procedures. Biointerface Res Appl Chem. 2019;9(6):4517-21.

156. Ke P-Y. Diverse functions of autophagy in liver physiology and liver diseases. Int J Mol Med Sci. 2019:20(2):300.

157. Yin X, Zhou C, Li J, Liu R, Shi B, Yuan Q, et al. Autophagy in bone homeostasis and the onset of osteoporosis. Bone Res. 2019:7(1):1-16.

158. Yang Y, Klionsky DJ. Autophagy and disease: unanswered questions. Cell Death Differ. 2020;27(3):858-71.

159. Ebrahimi-Fakhari D, Saffari A, Wahlster L, Lu J, Byrne S, Hoffmann GF, et al. Congenital disorders of autophagy: an emerging novel class of inborn errors of neuro-metabolism. Brain. 2016:139(2):317-37.

160. Ebrahimi-Fakhari D. Congenital disorders of autophagy: what a pediatric neurologist should know. Neuropediatrics. 2018;49(01):018-25.

161. Forlenza OV, De-Paula VDJR, Diniz BSO. Neuroprotective effects of lithium: implications for the treatment of Alzheimer's disease and related neurodegenerative disorders. ACS Chem Neurosci. 2014;5(6):443-50.

162. Brose RD, Lehrmann E, Zhang Y, Reeves RH, Smith KD, Mattson MP. Hydroxyurea attenuates oxidative, metabolic, and excitotoxic stress in rat hippocampal neurons and improves spatial memory in a mouse model of Alzheimer's disease. Neurobiol Aging. 2018;72:121-33.

163. Çelik H, Karahan H, Kelicen-Uğur P. Effect of atorvastatin on Aß1-42induced alteration of SESN2, SIRT1, LC3II and TPP1 protein expressions in neuronal cell cultures. J Pharm Pharmacol. 2020;72(3):424-36.

164. Phiwchai I, Chariyarangsitham W, Phatruengdet T, Pilapong C. Ferrictannic nanoparticles increase neuronal cellular clearance. ACS Chem Neurosci. 2019;10(9):4136-44.

165. Wang C, Zhang X, Teng Z, Zhang T, Li Y. Downregulation of PI3K/Akt/ mTOR signaling pathway in curcumin-induced autophagy in APP/PS1 double transgenic mice. Eur J Pharmacol. 2014;740:312-20.

166. Huang $M$, Jiang $X$, Liang $Y$, Liu Q, Chen S, Guo Y. Berberine improves cognitive impairment by promoting autophagic clearance and inhibiting production of $\beta$-amyloid in APP/tau/PS1 mouse model of Alzheimer's disease. Exp Gerontol. 2017:91:25-33.

167. Wang H, Jiang T, Li W, Gao N, Zhang T. Resveratrol attenuates oxidative damage through activating mitophagy in an in vitro model of Alzheimer's disease. Toxicol Lett. 2018;282:100-8.

168. Zhang Z, Wang X, Di Zhang YL, Li L. Geniposide-mediated protection against amyloid deposition and behavioral impairment correlates with downregulation of mTOR signaling and enhanced autophagy in a mouse model of Alzheimer's disease. Aging (Albany NY). 2019:11(2):536.

169. Wang X, Shan P, Liu A, Ma L, Lu M, Jiang W, et al. Polydatin prevents A beta-induced neuron cytotoxicity via enhancing autophagy and decreasing oxidative stress. Int J Clin Exp Med. 2017;10(11):15250-9.

170. Luengo E, Buendia I, Fernández-Mendívil C, Trigo-Alonso P, Negredo P, Michalska P, et al. Pharmacological doses of melatonin 
impede cognitive decline in tau-related Alzheimer models, once tauopathy is initiated, by restoring the autophagic flux. J Pineal Res. 2019;67(1):e12578.

171. Gugliandolo A, Chiricosta L, Silvestro S, Bramanti P, Mazzon E. a-Tocopherol modulates non-amyloidogenic pathway and autophagy in an in vitro model of Alzheimer's Disease: a transcriptional study. Brain Sci. 2019;9(8):196.

172. Rigacci S, Miceli C, Nediani C, Berti A, Cascella R, Pantano D, et al. Oleuropein aglycone induces autophagy via the AMPK/mTOR signalling pathway: a mechanistic insight. Oncotarget. 2015;6(34):35344.

173. Cordero JG, García-Escudero R, Avila J, Gargini R, García-Escudero V. Benefit of oleuropein aglycone for Alzheimer's disease by promoting autophagy. Oxid Med Cell Longev. 2018. https://doi.org/10.1155/2018/ 5010741.

174. Sharma C, Kang SC. Garcinol pacifies acrylamide induced cognitive impairments, neuroinflammation and neuronal apoptosis by modulating GSK signaling and activation of pCREB by regulating cathepsin B in the brain of zebrafish larvae. Food Chem Toxicol. 2020;138:111246.

175. Liu J, Su H, Qu Q-M. Carnosic acid prevents beta-amyloid-induced injury in human neuroblastoma sh-sy5y cells via the induction of autophagy. Neurochem Res. 2016;41(9):2311-23.

176. Xue Z, Guo Y, Zhang S, Huang L, He Y, Fang R, et al. Beta-asarone attenuates amyloid beta-induced autophagy via Akt/mTOR pathway in PC12 cells. Eur J Pharmacol. 2014;741:195-204

177. Yuan H, Jiang C, Zhao J, Zhao Y, Zhang Y, Xu Y, et al. Euxanthone Attenuates $A \beta$ 1-42-Induced Oxidative Stress and Apoptosis by Triggering Autophagy. J Mol Neurosci. 2018;66(4):512-23.

178. Al Rihani SB, Darakjian LI, Kaddoumi A. Oleocanthal-rich extra-virgin olive oil restores the blood-brain barrier function through NLRP3 inflammasome inhibition simultaneously with autophagy induction in TgSwDI mice. ACS Chem Neurosci. 2019;10(8):3543-54.

179. Ren Z-L, Wang C-D, Wang T, Ding H, Zhou M, Yang N, et al. Ganoderma lucidum extract ameliorates MPTP-induced parkinsonism and protects dopaminergic neurons from oxidative stress via regulating mitochondrial function, autophagy, and apoptosis. Acta Pharmacol Sin. 2019;40(4):441-50.

180. Sportelli C, Urso D, Jenner P, Chaudhuri K. Metformin as a potential neuroprotective agent in prodromal Parkinson's Disease. Front Neurol. 2020;11:556.

181. Zhou Q, Chen B, Wang X, Wu L, Yang Y, Cheng X, et al. Sulforaphane protects against rotenone-induced neurotoxicity in vivo: Involvement of the mTOR, Nrf2 and autophagy pathways. Sci Rep. 2016;6(1):1-12.

182. Wang B, Su C-J, Liu T-T, Zhou Y, Feng Y, Huang Y, et al. The neuroprotection of low-dose morphine in cellular and animal models of Parkinson's disease through ameliorating endoplasmic reticulum (ER) stress and activating autophagy. Front Mol Neurosci. 2018;11:120.

183. Ngwa HA, Kanthasamy A, Gu Y, Fang N, Anantharam V, Kanthasamy AG. Manganese nanoparticle activates mitochondrial dependent apoptotic signaling and autophagy in dopaminergic neuronal cells. Toxicol Appl Pharmacol. 2011;256(3):227-40.

184. Ding Y, Kong D, Zhou T, Xin C, Xu J, Wang Q, et al. a-Arbutin protects against Parkinson's disease-associated mitochondrial dysfunction in vitro and in vivo. Neuromol Med. 2020;22(1):56-67.

185. Bai H, Ding Y, Li X, Kong D, Xin C, Yang X, et al. Polydatin protects SHSY5Y in models of Parkinson's disease by promoting Atg5-mediated but parkin-independent autophagy. Neurochem Int. 2020;134:104671.

186. Yang G, Li J, Cai Y, Yang Z, Li R, Fu W. Glycyrrhizic acid alleviates 6-hydroxydopamine and corticosterone-induced neurotoxicity in SH-SY5Y cells through modulating autophagy. Neurochem Res. 2018;43(10):1914-26.

187. Zhou L, Cheng Y. Alpha-lipoic acid alleviated 6-OHDA-induced cell damage by inhibiting AMPK/mTOR mediated autophagy. Neuropharmacology. 2019;155:98-103.

188. Zeng R, Zhou Q, Zhang W, Fu X, Wu Q, Lu Y, et al. Icariin-mediated activation of autophagy confers protective effect on rotenone induced neurotoxicity in vivo and in vitro. Toxicol Rep. 2019;6:637-44.

189. Wei C-C, Chang C-H, Liao VHC. Anti-Parkinsonian effects of $\beta$-amyrin are regulated via LGG-1 involved autophagy pathway in Caenorhabditis elegans. Phytomedicine. 2017;36:118-25.

190. Filomeni G, Graziani I, De Zio D, Dini L, Centonze D, Rotilio G, et al. Neuroprotection of kaempferol by autophagy in models of rotenone-mediated acute toxicity: possible implications for Parkinson's disease. Neurobiol Aging. 2012;33(4):767-85.

191. Liu P, Li Y, Yang W, Liu D, Ji X, Chi T, et al. Prevention of Huntington's disease-like behavioral deficits in R6/1 mouse by tolfenamic acid is associated with decreases in mutant huntingtin and oxidative stress. Oxid Med Cell Longev. 2019. https://doi.org/10.1155/2019/4032428.

192. Chang C-C, Lin T-C, Ho H-L, Kuo C-Y, Li H-H, Korolenko TA, et al. GLP-1 analogue liraglutide attenuates mutant huntingtin-induced neurotoxicity by restoration of neuronal insulin signaling. Int J Mol Sci. 2018;19(9):2505.

193. Fernandez-Estevez MA, Casarejos MJ, López Sendon J, Garcia Caldentey J, Ruiz C, Gomez A, et al. Trehalose reverses cell malfunction in fibroblasts from normal and Huntington's disease patients caused by proteosome inhibition. PLoS ONE. 2014;9(2):e90202.

194. Vidoni C, Secomandi E, Castiglioni A, Melone MAB, Isidoro C. Resveratrol protects neuronal-like cells expressing mutant Huntingtin from dopamine toxicity by rescuing ATG4-mediated autophagosome formation. Neurochem Int. 2018;117:174-87.

195. Cordeiro LM, Machado ML, da Silva AF, Baptista FBO, da Silveira TL, Soares FAA, et al. Rutin protects Huntington's disease through the insulin/IGF1 (IIS) signaling pathway and autophagy activity: study in Caenorhabditis elegans model. Food Chem Toxicol. 2020;141:111323.

196. Yang J, Bridges K, Chen KY, Liu AYC. Riluzole increases the amount of latent HSF1 for an amplified heat shock response and cytoprotection. PLOS ONE. 2008;3(8):e2864.

197. Ueda T, Ito T, Kurita H, Inden M, Hozumi I. p-Coumaric acid has protective effects against mutant copper-zinc superoxide dismutase 1 via the activation of autophagy in N2a cells. Int J Mol Sci. 2019;20(12):2942.

198. Lv B, Jiang X-M, Wang D-W, Chen J, Han D-F, Liu X-L. Protective effects and mechanisms of action of ulinastatin against cerebral ischemiareperfusion injury. Curr Pharm Des. 2020;26(27):3332-40.

199. Wang R, Liu Y-Y, Liu X-Y, Jia S-W, Zhao J, Cui D, et al. Resveratrol protects neurons and the myocardium by reducing oxidative stress and ameliorating mitochondria damage in a cerebral ischemia rat model. Cell Physiol Biochem. 2014;34(3):854-64.

200. Yan BC, Wang J, Rui Y, Cao J, Xu P, Jiang D, et al. Neuroprotective effects of gabapentin against cerebral ischemia reperfusion-induced neuronal autophagic injury via regulation of the PI3K/Akt/mTOR signaling pathways. J Neuropathol Exp Neurol. 2019;78(2):157-71.

201. Yang B, Zang L-E, Cui J-W, Zhang M-Y, Ma X, Wei L-L. Melatonin plays a protective role by regulating miR-26a-5p-NRSF and JAK2-STAT3 pathway to improve autophagy, inflammation and oxidative stress of cerebral ischemia-reperfusion injury. Drug Des Devel Ther. 2020;14:3177.

202. Yu Y, Wu X, Pu J, Luo P, Ma W, Wang J, et al. Lycium barbarum polysaccharide protects against oxygen glucose deprivation/reoxygenationinduced apoptosis and autophagic cell death via the PI3K/Akt/mTOR signaling pathway in primary cultured hippocampal neurons. Biochem Biophys Res Commun. 2018;495(1):1187-94.

203. Yu F, Xue W, Dong L, Hu X, Huang D, Wang K. Tetrahydroxystilbene glucoside suppresses NAPDH oxidative stress to mitigate apoptosis and autophagy induced by cerebral ischemia/reperfusion injury in mice. Evid Based Complement Alternat Med. 2019. https://doi.org/10.1155/ 2019/3913981.

204. Yang H, Li L, Zhou K, Wang Y, Guan T, Chai C, et al. Shengmai injection attenuates the cerebral ischemia/reperfusion induced autophagy via modulation of the AMPK, mTOR and JNK pathways. Pharm Biol. 2016;54(10):2288-97.

205. Xu B, Zhu L, Chu J, Ma Z, Fu Q, Wei W, et al. Esculetin improves cognitive impairments induced by transient cerebral ischaemia and reperfusion in mice via regulation of mitochondrial fragmentation and mitophagy. Behav Brain Res. 2019;372:112007.

206. Wang N, He J, Pan C, Wang J, Ma M, Shi X, et al. Resveratrol activates autophagy via the AKT/mTOR signaling pathway to improve cognitive dysfunction in rats with chronic cerebral hypoperfusion. Front Neurosci. 2019;13:859.

207. Wojdasiewicz P, Poniatowski ŁA, Turczyn P, Frasuńska J, ParadowskaGorycka A, Tarnacka B. Significance of omega-3 fatty acids in the prophylaxis and treatment after spinal cord injury in rodent models. Mediators Inflamm. 2020. https://doi.org/10.1155/2020/3164260. 
208. Zhou KL, Chen DH, Jin HM, Wu K, Wang XY, Xu HZ, et al. Effects of calcitriol on experimental spinal cord injury in rats. Spinal Cord. 2016;54(7):510-6.

209. Wu Y-L, Chang J-C, Lin W-Y, Li C-C, Hsieh M, Chen H-W, et al. Caffeic acid and resveratrol ameliorate cellular damage in cell and Drosophila models of spinocerebellar ataxia type 3 through upregulation of Nrf2 pathway. Free Radic Biol Med. 2018;115:309-17.

210. Lin C-H, Wu Y-R, Yang J-M, Chen W-L, Chao C-Y, Chen I, et al. Novel lactulose and melibiose targeting autophagy to reduce polyQ aggregation in cell models of spinocerebellar ataxia 3. CNS Neurol Disord Drug Targets. 2016;15(3):351-9.

\section{Publisher's Note}

Springer Nature remains neutral with regard to jurisdictional claims in published maps and institutional affiliations.

- fast, convenient online submission

- thorough peer review by experienced researchers in your field

- rapid publication on acceptance

- support for research data, including large and complex data types

- gold Open Access which fosters wider collaboration and increased citations

- maximum visibility for your research: over $100 \mathrm{M}$ website views per year

At BMC, research is always in progress.

Learn more biomedcentral.com/submissions 